Homology, Homotopy and Applications, vol.5(1), 2003, pp.121-159

\title{
A THOM ISOMORPHISM FOR INFINITE RANK EUCLIDEAN BUNDLES
}

\author{
JODY TROUT \\ (communicated by Jonathan M. Rosenberg)
}

\begin{abstract}
An equivariant Thom isomorphism theorem in operator $K$ theory is formulated and proven for infinite rank Euclidean vector bundles over finite dimensional Riemannian manifolds. The main ingredient in the argument is the construction of a non-commutative $C^{\star}$-algebra associated to a bundle $\mathfrak{E} \rightarrow M$, equipped with a compatible connection $\nabla$, which plays the role of the algebra of functions on the infinite dimensional total space $\mathfrak{E}$. If the base $M$ is a point, we obtain the Bott periodicity isomorphism theorem of Higson-Kasparov-Trout [19] for infinite dimensional Euclidean spaces. The construction applied to an even finite rank spin'-bundle over an even-dimensional proper $\operatorname{spin}^{c}$-manifold reduces to the classical Thom isomorphism in topological $K$-theory. The techniques involve noncommutative geometric functional analysis.
\end{abstract}

\section{Introduction}

First, we review the classical Thom isomorphism in topological $K$-theory, in a smooth version appropriate for our infinite dimensional generalization. Let $M$ be a smooth finite dimensional manifold on which the compact group $G$ acts via diffeomorphisms. The equivariant topological $K$-theory group of $M$ (with compact supports) can be defined [36] as the abelian group $K_{G}^{0}(M)$ generated by $G$-homotopy equivalence classes $[\sigma]$ of smooth equivariant morphisms $\sigma: F_{1} \rightarrow F_{2}$ of smooth complex vector $G$-bundles (with finite rank) which are isomorphisms off a compact subset of $M$. Two such bundle morphisms $\sigma$ and $\sigma^{\prime}$ are $G$-homotopic if there is a $G$ bundle morphism $\Sigma$ on $M \times[0,1]$, also with compact support, such that $\left.\Sigma\right|_{M \times\{0\}} \cong \sigma$ and $\left.\Sigma\right|_{M \times\{1\}} \cong \sigma^{\prime}$. Addition in $K_{G}^{0}(M)$ is given by direct sum: $[\sigma]+\left[\sigma^{\prime}\right]=\left[\sigma \oplus \sigma^{\prime}\right]$. The map $M \mapsto K_{G}^{0}(M)$ defines a contravariant functor from the category of smooth $G$-manifolds (and smooth proper $G$-maps) to the category of abelian groups.

If $p: E \rightarrow M$ is a smooth vector $G$-bundle of finite rank on $M$ (either real or

The author was partially supported by NSF Grants DMS-9706767 and DMS-0071120 Received December 6, 2002, revised June 3, 2003; published on June 11, 2003.

2000 Mathematics Subject Classification: 19, 46, 47, 55, 57, 58

Key words and phrases: Thom isomorphism, operator algebras, K-theory, vector bundles (C) 2003, Jody Trout. Permission to copy for private use granted. 
complex), then $K_{G}^{0}(E)$ has a right $K_{G}^{0}(M)$-module structure via the composition

$$
K_{G}^{0}(E) \times K_{G}^{0}(M) \stackrel{\stackrel{\otimes}{\longrightarrow}}{\longrightarrow} K_{G}^{0}(E \times M) \stackrel{(\mathrm{id} \times p)^{*}}{\longrightarrow} K_{G}^{0}(E)
$$

where the first homomorphism is given by the outer tensor product $\nabla$ and the second homomorphism is induced by the proper $G$-map id $\times p: E \rightarrow E \times M$. Give the vector bundle $E$ a $G$-invariant bundle metric $(\cdot, \cdot)$ and let

$$
\operatorname{Cliff}\left(p^{*} E\right)=\operatorname{Cliff}\left(p^{*} E\right)_{\mathrm{ev}} \oplus \operatorname{Cliff}\left(p^{*} E\right)_{\mathrm{od}}
$$

denote the complex Clifford algebra bundle [4] of the pullback $p^{*} E \rightarrow E$ with its natural $\mathbb{Z}_{2}$-grading and $G$-action. Consider the smooth $G$-equivariant morphism

$$
C_{E}: \operatorname{Cliff}\left(p^{*} E\right)_{\mathrm{ev}} \rightarrow \operatorname{Cliff}\left(p^{*} E\right)_{\mathrm{od}}
$$

which is defined via Clifford multiplication by the basepoint

$$
C_{E}(\omega)(e)=e \cdot \omega, \quad e \in E, \omega \in \operatorname{Cliff}\left(E_{p(e)}\right)_{\mathrm{ev}} .
$$

It is a vector bundle isomorphism off the zero section $M \subset E$. If $M$ is compact, this defines a $K$-theory element, called the Thom class,

$$
\lambda_{E}=\left[C_{E}: \operatorname{Cliff}\left(p^{*} E\right)_{\mathrm{ev}} \rightarrow \operatorname{Cliff}\left(p^{*} E\right)_{\mathrm{od}}\right] \in K_{G}^{0}(E) .
$$

The Thom homomorphism is then defined as the mapping

$$
\Phi: K_{G}^{0}(M) \rightarrow K_{G}^{0}(E):[\sigma] \mapsto \lambda_{E} \otimes[\sigma]
$$

via module multiplication by the Thom class. If $M$ is not compact, then $\lambda_{E}$ does not define a $K$-theory class for $E$, but multiplication by $\lambda_{E}$ is still well-defined since $\lambda_{E} \otimes[\sigma]$ has compact support and we obtain a similar Thom homomorphism in the non-compact case. An important result in topological $K$-theory is the Thom isomorphism theorem $[\mathbf{3}, \mathbf{2 2}, \mathbf{3 6}]$ which is the statement that if $p: E \rightarrow M$ is an oriented Euclidean vector bundle (of finite even rank) with spin ${ }^{c}$-structure then $\Phi: K_{G}^{0}(M) \rightarrow K_{G}^{0}(E)$ is an isomorphism of abelian groups.

Recall that if we take $M=\{\bullet\}$ to be a point, then $E \cong \mathbb{R}^{2 n}$ is a finite dimensional Euclidean vector space and the Thom isomorphism of $\mathbb{R}^{2 n} \rightarrow\{\bullet\}$ is the Bott periodicity isomorphism $K_{G}^{0}(\{\bullet\}) \cong K_{G}^{0}\left(\mathbb{R}^{2 n}\right)$. The Thom isomorphism can then be viewed, in general, as multiplication by the Bott element $\lambda_{\mathbb{R}^{2 n}}$ along the fibers of $E \rightarrow M$, where $\operatorname{rank}(E)=2 n$.

If a vector bundle $p: \mathfrak{E} \rightarrow M$ has infinite rank, say, if $\mathfrak{E}$ is a real (or complex) Hilbert bundle with infinite dimensional fiber $\mathcal{E}$, then none of this works. This is because the total space $\mathfrak{E}$ is is not locally compact, and so lies outside the scope of ordinary topological $K$-theory (even for compact groups.) We could try using an equivariant Swan's Theorem $[\mathbf{3}, \mathbf{2 9}] K_{G}^{0}(M) \cong K_{0}^{G}\left(C_{0}(M)\right)$, where $C_{0}(M)$ is the $C^{\star}$-algebra of continuous complex-valued functions on $M$ which vanish at infinity. However, this attempt fails because $\mathfrak{E}$ is infinite dimensional and so $C_{0}(\mathfrak{E})=\{0\}$. Another problem occurs when trying to define an equivariant $K$-theory when the transformation group $G$ is non-compact, since using finite rank vector $G$-bundles is no longer appropriate. However, N. C. Phillips [30] has defined a topological 
equivariant $K$-theory $K_{G}^{0}(M)$ for proper actions of locally compact groups. He then proves a generalized Green-Julg-Rosenberg isomorphism:

$$
K_{G}^{0}(M) \cong K_{0}\left(C_{0}(M) \rtimes_{\alpha} G\right)
$$

where $C_{0}(M) \rtimes_{\alpha} G$ is (any) crossed product of $A$ by $G$. But, again we have the problem that $\mathfrak{E}$ is not locally compact. Most importantly, non-compact groups do not act properly on a point. Thus, we must appeal directly to inherently non-commutative geometric methods.

In this paper, we remedy these problems by defining an appropriate (equivariant) Thom homomorphism using the $K$-theory of non-commutative $C^{\star}$ - algebras. The method is suggested by considering the case when $M=\{\bullet\}$ is a point and so $\mathfrak{E}=\mathcal{E}$ is an infinite dimensional Euclidean space. In this very important case, we should obtain the Bott periodicity of Higson-Kasparov-Trout [19]. Let $G$ be a smooth, second countable, locally compact Hausdorff topological group, e.g. a countable discrete group.

Let $\mathcal{E}$ be a Euclidean (i.e., real Hilbert) space of countably infinite dimension. If $E_{a}$ is a finite dimensional subspace of $\mathcal{E}$, then denote by $\mathcal{C}\left(E_{a}\right)$ the $C^{\star}$-algebra $C_{0}\left(E_{a}, \operatorname{Cliff}\left(E_{a}\right)\right)$ of continuous functions from $E_{a}$ into the complexified Clifford algebra of $E_{a}$ vanishing at infinity. This $C^{\star}$-algebra has a $\mathbb{Z}_{2}$-grading inherited from $\operatorname{Cliff}\left(E_{a}\right)$. Let $\mathcal{A}\left(E_{a}\right)$ denote the $\mathbb{Z}_{2}$-graded tensor product,

$$
\mathcal{A}\left(E_{a}\right)=C_{0}(\mathbb{R}) \hat{\otimes} \mathcal{C}\left(E_{a}\right),
$$

where $C_{0}(\mathbb{R})$ is graded into even and odd functions. If $E_{a}$ is a subspace of another finite dimensional subspace $E_{b}$ of $\mathcal{E}$, then there is a canonical homomorphism of $C^{\star}$-algebras $\mathcal{A}\left(E_{a}\right) \rightarrow \mathcal{A}\left(E_{b}\right)$ (described in more generality in Section 2.) These homomorphisms are injective and, more surprisingly, are functorial with respect to subspace inclusion. This allows us to define the direct limit $C^{\star}$-algebra

$$
\mathcal{A}(\mathcal{E})=\underset{E_{a} \subset \mathcal{E}}{\underset{\lim }{\longrightarrow}} \mathcal{A}\left(E_{a}\right)
$$

taken over the directed system of all finite dimensional linear subspaces $\left\{E_{a}\right\}$ of the infinite dimensional Euclidean space $\mathcal{E}$. If $\mathcal{E}$ is equipped with an orthogonal action of the group $G$, then $G$ acts on the directed system of subspaces, so it induces an action on the direct limit. Most importantly, the equivariant inclusion of the zero dimensional subspace $\{0\} \hookrightarrow \mathcal{E}$ induces a canonical equivariant $*$-homomorphism $\beta: \mathcal{A}(0) \rightarrow \mathcal{A}(\mathcal{E})$. The Bott periodicity theorem of Higson-Kasparov-Trout [19] is the statement that the map $\beta: \mathcal{A}(0) \rightarrow \mathcal{A}(\mathcal{E})$ induces an isomorphism in equivariant $K$-theory $^{1}$ :

$$
\beta_{*}: K_{*}^{G}(\mathcal{A}(0)) \rightarrow K_{*}^{G}(\mathcal{A}(\mathcal{E})) .
$$

Since $\mathcal{A}(0) \rtimes G \cong C_{0}(\mathbb{R}) \otimes C^{*} G$ it follows that $K_{*}^{G}(\mathcal{A}(0)) \cong K_{*+1}\left(C^{*} G\right)$, where $C^{*} G$ is the (full) group $C^{\star}$-algebra of $G$. Compare this to the compact group case where $K_{G}^{0}(\{\bullet\})=R(G) \cong K_{0}\left(C^{*} G\right)$ by the Peter-Weyl Theorem, where $R(G)$ is the representation ring of the compact group $G$.

${ }^{1}$ We define $K_{*}^{G}(A)=K_{*}(A \rtimes G)$ where $A \rtimes G$ is the full crossed product (see Definition 4.4). 
These considerations suggest the following. Let $M$ be a finite dimensional Riemannian manifold. Let $\mathcal{C}(M)$ denote the $C^{\star}$-algebra of continuous sections of the complexified Clifford algebra bundle Cliff $(T M)$ of the tangent bundle $T M$ vanishing at infinity. Let

$$
\mathcal{A}(M)=C_{0}(\mathbb{R}) \hat{\otimes} \mathcal{C}(M)
$$

denote the $\mathbb{Z}_{2}$-graded suspension of $\mathcal{C}(M)$. These non-commutative $C^{\star}$-algebras will play a fundamental role in defining our Thom homomorphism for infinite rank Euclidean bundles. If $M$ is an oriented even-dimensional $\operatorname{spin}^{c}$-manifold, there is a Morita equivalence [34] (given by the spinor bundle) between $\mathcal{C}(M)$ and $C_{0}(M)$. It follows that if the group $G$ acts properly on $M$ in a compatible way:

$$
K_{*}^{G}(\mathcal{A}(M)) \cong K_{*+1}\left(C_{0}(M) \rtimes G\right) \cong K_{G}^{*+1}(M) .
$$

However, a non-compact group does not act properly on a point. Thus, we must allow for a more general class of group actions, called isoreductive actions (see Definition 4.1) which have a local slice property similar to the notion of proper action as given by Baum-Connes-Higson [6].

Now let $p: \mathfrak{E} \rightarrow M$ be a smooth Euclidean $G$-bundle modelled on $\mathcal{E}$, i.e., a locally trivial fiber bundle with fiber given by a Euclidean space $\mathcal{E}$, of countably infinite dimension, and structure group $O(\mathcal{E})$ in the norm (or strong) operator topology. We assume that the group $G$ acts by Euclidean bundle automorphisms of $\mathfrak{E}$ and acts on $M$ isoreductively. We will generalize the $C^{\star}$-algebra construction above to obtain a direct limit $C^{\star}$-algebra

$$
\mathcal{A}(\mathfrak{E})=\underset{E^{a} \subset \mathfrak{E}}{\lim _{\vec{m}}} \mathcal{A}\left(E^{a}\right)
$$

taken over the directed system of all smooth finite rank Euclidean subbundles $\mathfrak{E} \supset E^{a} \rightarrow M$ ordered by inclusion of subbundles. In order for this construction to succeed, we must equip $\mathfrak{E}$ with a compatible $G$-invariant connection $\nabla$ which controls splittings used in defining the connecting maps of the direct limit. The equivariant inclusion of $M \hookrightarrow \mathfrak{E}$ as the zero subbundle then canonically induces an equivariant "Thom" $*$-homomorphism $\Psi_{p}: \mathcal{A}(M) \rightarrow \mathcal{A}(\mathfrak{E})$. The induced map on equivariant $K$-theory $\Psi_{*}^{G}: K_{*}^{G}(\mathcal{A}(M)) \rightarrow K_{*}^{G}(\mathcal{A}(\mathfrak{E}))$ is our desired equivariant Thom homomorphism, and our main result is this:

Theorem 1.1. Let $G$ be a smooth, second countable, locally compact group and $M$ be a smooth isoreductive Riemannian $G$-manifold. If $p: \mathfrak{E} \rightarrow M$ is a Euclidean $G$-bundle with fiber a countably infinite dimensional Euclidean space $\mathcal{E}$, equipped with a $G$-invariant connection, then the map $\Psi_{*}^{G}: K_{*}^{G}(\mathcal{A}(M)) \rightarrow K_{*}^{G}(\mathcal{A}(\mathfrak{E}))$ is an isomorphism of abelian groups.

The outline is as follows. In Section 2, we develop the non-commutative geometric tools needed to define our infinite rank Thom homomorphism and discuss the relations with the classical finite rank case. In Section 3, we construct the $C^{\star}$-algebra $\mathcal{A}(\mathfrak{E})$ and prove the non-equivariant version of Theorem 1.1. The equivariant Thom homomorphism is developed in Section 4 and the proof of Theorem 1.1 is given in Section 5. In Appendix A we review $\mathbb{Z}_{2}$-graded $C^{\star}$-algebras and their (essentially) 
self-adjoint unbounded multipliers and introduce a new balanced tensor product for $\mathbb{Z}_{2}$-graded $C_{0}(M)$-algebras that we need to make our construction work.

The author would like to thank P. Baum, N. Higson, D. Williams, I. Raeburn, C. Gordon, N. C. Phillips, E. Guentner, and D. Dumitrascu for helpful suggestions and enlightening discussions.

\section{Clifford $C^{\star}$-algebras and Thom Isomorphism: The Finite Rank Case}

We assume throughout this section that all manifolds are finite dimensional, second countable, Hausdorff, and infinitely differentiable, and all maps are infinitely smooth. Let $M$ be a Riemannian manifold with tangent bundle $T M \rightarrow M$.

Definition 2.1. Let Cliff $(T M)$ denote the Clifford bundle [4, 7] of TM. That is, the bundle of Clifford algebras over $M$ whose fiber at $x \in M$ is the complex Clifford algebra $\operatorname{Cliff}\left(T_{x} M\right)$ of the Euclidean space $T_{x} M$, i.e., the universal unital complex *-algebra that contains $T_{x} M$ as a real linear subspace such that $v_{x}^{2}=\left\|v_{x}\right\|^{2} 1$ for all $v_{x} \in T_{x} M$. It has a natural $\mathbb{Z}_{2}$-graded Hermitian bundle structure.

Recall that the complex Clifford algebra of a finite dimensional Euclidean space has a canonical $C^{\star}$-algebra structure $[\mathbf{1 9}, \mathbf{2 2}, \mathbf{2 1}]$.

Definition 2.2. Denote by $\mathcal{C}(M)$ the $C^{\star}$-algebra $C_{0}(M$, Cliff $(T M))$ of continuous sections of $\operatorname{Cliff}(T M)$ which vanish at infinity, with $\mathbb{Z}_{2}$-grading induced from Cliff $(T M)$. (See Appendix A for a review of graded $C^{\star}$-algebras.)

To be precise, we should use the notation $\mathcal{C}(M, g)$ to show the dependence of the $C^{\star}$-algebra $\mathcal{C}(M)$ on the Riemannian metric $g$ of $M$. However, any two bundle metrics $g_{1}$ and $g_{2}$ on $T M$ are equivalent via a Euclidean bundle isomorphism $\left(T M, g_{1}\right) \cong\left(T M, g_{2}\right)$ which, by universality, induces a unitary $\mathbb{Z}_{2}$-graded bundle isomorphism Cliff $\left(T M, g_{1}\right) \cong \operatorname{Cliff}\left(T M, g_{2}\right)$ of Clifford algebra bundles. Thus, the $C^{\star}$-algebras $\mathcal{C}\left(M, g_{1}\right) \cong \mathcal{C}\left(M, g_{2}\right)$ are isomorphic as $\mathbb{Z}_{2}$-graded $C^{\star}$-algebras.

Let $C_{0}(M)$ denote the $C^{\star}$-algebra of continuous complex-valued functions on $M$ which vanish at infinity. Note that pointwise multiplication

$$
(f s)(x)=f(x) s(x), \quad \forall x \in M,
$$

where $f \in C_{0}(M)$ and $s \in \mathcal{C}(M)$, determines a nondegenerate $*$-homomorphism

$$
C_{0}(M) \rightarrow Z M(\mathcal{C}(M))
$$

into the center of the multiplier algebra of $\mathcal{C}(M)$ of grading degree zero (where $C_{0}(M)$ is always considered to be trivially graded.) Thus, we have the following.

Lemma 2.3. The $C^{\star}$-algebra $\mathcal{C}(M)$ has a canonical $C_{0}(M)$-algebra structure, and up to $\mathbb{Z}_{2}$-graded isomorphism, is independent of the Riemannian metric on $M$.

If $M=V$ is a finite dimensional Euclidean vector space, then $T M=V \times V$ and so $\mathcal{C}(M)=C_{0}(V, \operatorname{Cliff}(V))$ as in Definition $2.2[\mathbf{1 9}]$. Using the canonical isomorphism $T\left(M_{1} \times M_{2}\right) \cong T M_{1} \times T M_{2}$, we easily obtain the following result. 
Lemma 2.4. If $M=M_{1} \times M_{2}$ then $\mathcal{C}(M) \cong \mathcal{C}\left(M_{1}\right) \hat{\otimes} \mathcal{C}\left(M_{2}\right)$.

Definition 2.5. Let $\mathcal{S}$ denote the $C^{\star}$-algebra $C_{0}(\mathbb{R})$ of continuous complex-valued functions on the real line which vanish at infinity, graded into even and odd functions. If $A$ is any $\mathbb{Z}_{2}$-graded $C^{\star}$-algebra then let $\mathcal{S} A$ be the graded tensor product $\mathcal{S} \hat{\otimes} A$. In particular, let $\mathcal{A}(M)=\mathcal{S} \hat{\otimes} \mathcal{C}(M)$, which can be viewed as a noncommutative topological suspension ${ }^{2}$ of $M$.

Another relationship between the non-commutative $C^{\star}$-algebra $\mathcal{C}(M)$ and the commutative $C^{\star}$-algebra $C_{0}(M)$ is given by $\operatorname{spin}^{c}$-structures $[\mathbf{2 1}]$. Let $\mathbb{C}_{1}=\operatorname{Cliff}(\mathbb{R})$ denote the first complex Clifford algebra. The following is adapted from Theorem 2.11 of Plymen [31] and Proposition II.A.9 of Connes [12].

Proposition 2.6. Let $M$ be an oriented Riemannian manifold. If $M$ is evendimensional, there is a bijective correspondence between spin ${ }^{c}$-structures on $M$ and Morita equivalences (in the sense of Rieffel [34, 35]) between the $C^{\star}$-algebras $C_{0}(M)$ and $\mathcal{C}(M)$. Thus, $\mathcal{A}(M)$ is Morita equivalent to $C_{0}(\mathbb{R} \times M)$. If $M$ is odddimensional, then spin ${ }^{c}$-structures on $M$ are in bijective correspondence with Morita equivalences $C_{0}(M) \sim \mathcal{C}(M) \hat{\otimes} \mathbb{C}_{1}$.

Proof. Assume $\operatorname{dim}(M)=2 m$ is even. Let $S \rightarrow M$ denote the spinor bundle associated to a $\operatorname{spin}^{c}$-structure. This has a natural Euclidean bundle structure. The module $\mathcal{A}_{S}=C_{0}(M, S)$ of continuous sections of $S$ vanishing at infinity gives the desired $C_{0}(M)-\mathcal{C}(M)$-imprimitivity bimodule implementing the Morita equivalence $C_{0}(M) \sim \mathcal{C}(M)$. Since $M$ is even-dimensional,

$$
\operatorname{Cliff}(T M)=\operatorname{Cliff}(T M)_{\mathrm{ev}} \oplus \operatorname{Cliff}(T M)_{\mathrm{od}}
$$

splits as a $\mathbb{Z}_{2}$-bundle with grading operator given by the bounded global section

$$
\varepsilon=i^{m} e_{1} \cdot e_{2} \cdots e_{2 m}
$$

where $\left\{e_{1}, \cdots, e_{2 m}\right\}$ is any oriented local orthonormal frame of $T M$. It follows that $\mathcal{C}(M)=C_{0}(M, \operatorname{Cliff}(T M))$ is an evenly graded $C^{\star}$-algebra with grading operator $\varepsilon \in M(\mathcal{C}(M))$. Thus, by Proposition 14.5.1 [8], the graded tensor product $C_{0}(\mathbb{R}) \hat{\otimes} \mathcal{C}(M)$ and ungraded tensor product $C_{0}(\mathbb{R}) \otimes \mathcal{C}(M)$ are isomorphic as (ungraded) $C^{\star}$-algebras. Thus, since suspensions preserve Morita equivalence:

$$
\mathcal{A}(M)={ }_{\text {def }} C_{0}(\mathbb{R}) \hat{\otimes} \mathcal{C}(M) \cong C_{0}(\mathbb{R}) \otimes \mathcal{C}(M) \sim C_{0}(\mathbb{R}) \otimes C_{0}(M) \cong C_{0}(\mathbb{R} \times M) .
$$

The odd-dimensional case is similar, but we cannot remove the grading on the suspension and we must tensor by $\mathbb{C}_{1}$.

Although $\mathcal{C}(M)$ and $\mathcal{A}(M)$ carry natural $\mathbb{Z}_{2}$-gradings, when we consider their $K$-theory, we shall ignore the gradings. To make this point clearer, if $A$ is any $C^{\star}$-algebra-graded or not-then $K_{*}(A)$ will denote the $K$-theory of the underlying $C^{\star}$-algebra, without the grading.

Corollary 2.7. If $M$ is an even-dimensional oriented Riemannian manifold with spin ${ }^{\text {- }}$-structure, there are canonical $K$-theory isomorphisms

$$
K_{*}(\mathcal{C}(M)) \cong K^{*}(M) \text { and } K_{*}(\mathcal{A}(M)) \cong K^{*+1}(M) .
$$

${ }^{2}$ Recall that for the ungraded tensor product we have $C_{0}(\mathbb{R}) \otimes C_{0}(M) \cong C_{0}(\mathbb{R} \times M)$. 
Let $p: E \rightarrow M$ be a smooth finite rank Euclidean bundle. We wish to show that there is a natural "Thom" $*$-homomorphism

$$
\Psi_{p}: \mathcal{A}(M) \rightarrow \mathcal{A}(E)
$$

where we consider $E$ as a finite dimensional manifold with Riemannian structure to be constructed as follows.

Given $p: E \rightarrow M$, there is a short exact sequence $[2,7]$ of real vector bundles

$$
0 \longrightarrow V E \longrightarrow T E \stackrel{T^{*} p}{\longrightarrow} p^{*} T M \longrightarrow 0
$$

where the vertical subbundle $V E=\operatorname{ker}\left(T^{*} p\right)$ is isomorphic to $p^{*} E$. This sequence does not have a canonical splitting, in general, but choosing a compatible connection $\nabla$ on $E$ determines an associated vector bundle splitting.

Definition 2.8. A connection [7, 21] on $E$ is a linear map

$$
\nabla: C^{\infty}(M, E) \rightarrow C^{\infty}\left(M, T^{*} M \otimes E\right)
$$

which satisfies Leibnitz's rule, i.e., if $s \in C^{\infty}(M, E)$ and $f \in C^{\infty}(M)$, then

$$
\nabla(f s)=d f \otimes s+f \nabla s
$$

where $d$ is the exterior derivative on smooth forms $\Omega^{*}(M)=C^{\infty}\left(M, \Lambda^{*} T^{*} M\right)$. If $E$ has a Euclidean metric $(\cdot, \cdot)$, we say that $\nabla$ is compatible with the metric if

$$
X\left(s_{1}, s_{2}\right)=\left(\nabla_{X} s_{1}, s_{2}\right)+\left(s_{1}, \nabla_{X} s_{2}\right)
$$

for all sections $s_{1}, s_{2} \in C^{\infty}(M, E)$ and vector fields $X \in C^{\infty}(M, T M)$. If $E$ is equipped with a compatible connection $\nabla$, then we call $E$ an affine Euclidean bundle.

Lemma 2.9. Let $p: E \rightarrow M$ be a finite rank Euclidean bundle on the Riemannian manifold $M$. Given a compatible connection $\nabla$ on $E$ there is an induced orthogonal splitting $T E \cong p^{*} E \oplus p^{*} T M$ of the exact sequence

$$
0 \rightarrow p^{*} E \rightarrow T E \rightarrow p^{*} T M \rightarrow 0
$$

where $p^{*} E$ and $p^{*} T M$ have the pullback metrics. Hence, the manifold $E$ has an induced Riemannian metric.

Proof. Let $\nabla^{*}: C^{\infty}\left(E, p^{*} E\right) \rightarrow C^{\infty}\left(T^{*} E \otimes p^{*} E\right)$ denote the pullback of $\nabla$ on the bundle $p^{*} E \rightarrow E$, which is defined by the formula:

$$
\nabla^{*}\left(f p^{*} s\right)=d f \otimes p^{*} s+f p^{*}(\nabla s)
$$

for $f \in C^{\infty}(M)$ and $s \in C^{\infty}(M, E)$. The tautological section $\tau \in C^{\infty}\left(E, p^{*} E\right)$ is the smooth section defined by the formula $\tau(e)=(e, e)$ for all $e \in E$. The derivative of $\tau$ will be denoted by

$$
\omega=\nabla^{*} \tau \in C^{\infty}\left(T^{*} E \otimes p^{*} E\right)=\Omega^{1}\left(E, p^{*} E\right) \cong \Omega^{1}(E, V E)
$$

which is a connection 1-form (Definition $1.10[7]$ ). The kernel $H E=\operatorname{ker}(\omega) \cong p^{*} T M$ of the connection 1-form $\omega$ is the horizontal subbundle of $T E$ which provides the desired splitting

$$
T E=V E \oplus H E \cong p^{*} E \oplus p^{*} T M .
$$


Now give $T E$ the direct sum of the pullback metrics on $p^{*} E$ and $p^{*} T M$. This gives $E$ the structure of a Riemannian manifold and makes the splitting of $T E$ orthogonal.

Thus, given a compatible connection $\nabla$ on the Euclidean bundle $E$, we can define the $C^{\star}$-algebra $\mathcal{C}(E)$ as above using the induced Riemannian structure on the manifold $E$. However, we also have the $C^{\star}$-algebra $C_{0}\left(E, \operatorname{Cliff}\left(p^{*} E\right)\right)$ associated to the pullback bundle $p^{*} E \rightarrow E$. Both $\mathcal{C}(E)$ and $C_{0}\left(E, \operatorname{Cliff}\left(p^{*} E\right)\right)$ have natural $C_{0}(E)$-algebra structures. However, the bundle map $p: E \rightarrow M$ induces a pullback *-homomorphism [33]

$$
p^{*}: C_{0}(M) \rightarrow C_{b}(E)=M\left(C_{0}(E)\right): f \mapsto p^{*}(f)=f \circ p
$$

which induces a (graded) $C_{0}(M)$-algebra structure on any (graded) $C_{0}(E)$-algebra. The following is an important result that relates these two $C^{\star}$-algebras ${ }^{3}$ to the $C_{0}(M)$-algebra $\mathcal{C}(M)$ of the base manifold $M$.

Theorem 2.10. Let $p: E \rightarrow M$ be a finite rank affine Euclidean bundle on the Riemannian manifold $M$. There is a natural isomorphism of graded $C^{\star}$-algebras

$$
\mathcal{C}(E) \cong C_{0}\left(E, \operatorname{Cliff}\left(p^{*} E\right)\right) \hat{\otimes}_{M} \mathcal{C}(M)
$$

where $\hat{\otimes}_{M}$ denotes the balanced tensor product over $C_{0}(M)$ of Definition A.6.

Proof. By the previous lemma, there is an induced orthogonal splitting

$$
T E=p^{*} E \oplus p^{*} T M
$$

Thus, we have an induced isomorphism of $\mathbb{Z}_{2}$-graded Clifford algebra bundles

$$
\operatorname{Cliff}(T E) \cong \operatorname{Cliff}\left(p^{*} E \oplus p^{*} T M\right)=\operatorname{Cliff}\left(p^{*} E\right) \hat{\otimes} p^{*} \operatorname{Cliff}(T M) .
$$

Therefore, by taking sections, we have canonical balanced tensor product isomorphisms (see Proposition A.7)

$$
\begin{aligned}
\mathcal{C}(E) & ={ }_{\operatorname{def}} C_{0}(E, \operatorname{Cliff}(T E)) \cong C_{0}\left(E, \operatorname{Cliff}\left(p^{*} E\right) \hat{\otimes} p^{*} \operatorname{Cliff}(T M)\right) \\
& \cong C_{0}\left(E, \operatorname{Cliff}\left(p^{*} E\right)\right) \hat{\otimes}_{E} C_{0}\left(E, \operatorname{Cliff}\left(p^{*} T M\right)\right) .
\end{aligned}
$$

But, we have, using pullbacks along $p: E \rightarrow M$, that there are canonical pullback isomorphisms (Proposition A.9)

$$
C_{0}\left(E, \operatorname{Cliff}\left(p^{*} T M\right)\right) \cong p^{*} C_{0}(M, \operatorname{Cliff}(T M))=p^{*} \mathcal{C}(M)={ }_{\operatorname{def}} C_{0}(E) \hat{\otimes}_{M} \mathcal{C}(M) .
$$

Hence, it follows that

$$
\begin{aligned}
\mathcal{C}(E) \cong & C_{0}\left(E, \operatorname{Cliff}\left(p^{*} E\right)\right) \hat{\otimes}_{E} C_{0}\left(E, \operatorname{Cliff}\left(p^{*} T M\right)\right) \\
& \cong C_{0}\left(E, \operatorname{Cliff}\left(p^{*} E\right)\right) \hat{\otimes}_{E} C_{0}(E) \hat{\otimes}_{M} \mathcal{C}(M) \\
& \cong C_{0}\left(E, \operatorname{Cliff}\left(p^{*} E\right)\right) \hat{\otimes}_{M} \mathcal{C}(M)
\end{aligned}
$$

using the canonical isomorphism $A \hat{\otimes}_{E} C_{0}(E) \cong A$ for graded $C_{0}(E)$-algebras.

We now wish to define a certain "Thom operator" for the "vertical" algebra $C_{0}\left(E, \operatorname{Cliff}\left(p^{*} E\right)\right)$.

${ }^{3}$ Although $C_{0}\left(E, \operatorname{Cliff}\left(p^{*} E\right)\right) \cong p^{*} C_{0}(M, \operatorname{Cliff}(E))$, we will not need this isomorphism. 
Associate to the Euclidean bundle $E$ an unbounded section

$$
C_{E}: E \rightarrow \operatorname{Cliff}\left(p^{*} E\right): e \mapsto C_{p(e)}(e)
$$

where $C_{p(e)}$ is the Clifford operator on the Euclidean space $E_{p(e)}$ from Definition $2.4[\mathbf{1 9}]$. It is given globally by the composition

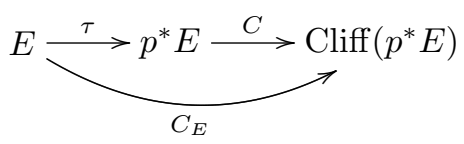

where $\tau \in C^{\infty}\left(E, p^{*} E\right)$ is the tautological section (in the proof of Lemma 2.9) and $C: p^{*} E \hookrightarrow \operatorname{Cliff}\left(p^{*} E\right)$ is the canonical inclusion $C\left(e_{1}, e_{2}\right)=C_{p\left(e_{1}\right)}\left(e_{2}\right)$. The following is then easy to prove.

Theorem 2.11. Let $E$ be a finite rank Euclidean bundle on $M$. Multiplication by the section $C_{E}: E \rightarrow \operatorname{Cliff}\left(p^{*} E\right)$ determines a degree one, essentially self-adjoint, unbounded multiplier (see Definition A.1) of the $C^{\star}$-algebra $C_{0}\left(E, \operatorname{Cliff}\left(p^{*} E\right)\right)$ with domain $C_{c}\left(E, \operatorname{Cliff}\left(p^{*} E\right)\right)$.

We will call $C_{E}$ the Thom operator of $E \rightarrow M$. Thus, we have a functional calculus homomorphism

$$
\mathcal{S}=C_{0}(\mathbb{R}) \rightarrow M\left(C_{0}\left(E, \operatorname{Cliff}\left(p^{*} E\right)\right)\right): f \rightarrow f\left(C_{E}\right)
$$

from $\mathcal{S}$ to the multiplier algebra of $C_{0}\left(E, \operatorname{Cliff}\left(p^{*} E\right)\right)$. Note that $f\left(C_{E}\right)$ goes to zero in the "fiber" directions on $E$ (since $p(e)$ is constant), but is only bounded in the "manifold" directions on $E$. Indeed, note that for the generators $f(x)=\exp \left(-x^{2}\right)$ and $g(x)=x \exp \left(-x^{2}\right)$ of $\mathcal{S}$, we have that $f\left(C_{E}\right)$ and $g\left(C_{E}\right)$ are, respectively, multiplication by the following functions on $E$ :

$$
f\left(C_{E}\right)(e)=\exp \left(-\|e\|^{2}\right) \text { and } g\left(C_{E}\right)(e)=e \cdot \exp \left(-\|e\|^{2}\right), \quad \forall e \in E .
$$

Definition 2.12. Let $X$ denote the degree one, essentially self-adjoint, unbounded multiplier of $\mathcal{S}=C_{0}(\mathbb{R})$, with domain the compactly supported functions, given by multiplication by $x$, i.e., $X f(x)=x f(x)$ for all $f \in C_{c}(\mathbb{R})$ and $x \in \mathbb{R}$.

By Lemma A.3, the operator $X \hat{\otimes} 1+1 \hat{\otimes} C_{E}$ determines a degree one, essentially self-adjoint, unbounded multiplier of the tensor product

$$
\mathcal{S} \hat{\otimes} C_{0}\left(E, \operatorname{Cliff}\left(p^{*} E\right)\right)=\mathcal{S} C_{0}\left(E, \operatorname{Cliff}\left(p^{*} E\right)\right) .
$$

We obtain a functional calculus homomorphism

$$
\beta_{E}: \mathcal{S} \rightarrow M\left(\mathcal{S} C_{0}\left(E, \operatorname{Cliff}\left(p^{*} E\right)\right)\right): f \mapsto f\left(X \hat{\otimes} 1+1 \hat{\otimes} C_{E}\right)
$$

from $\mathcal{S}$ into the multiplier algebra of $\mathcal{S} C_{0}\left(E, \operatorname{Cliff}\left(p^{*} E\right)\right)$. Now we can define our "Thom $*$-homomorphism" for a finite rank affine Euclidean bundle. This will provide the connecting map in the next section to define the direct limit $C^{\star}$-algebra for an infinite rank affine Euclidean bundle.

Theorem 2.13. Let $(E, \nabla, M)$ be as above. With respect to the isomorphism

$$
\mathcal{A}(E) \cong \mathcal{S} \hat{\otimes} C_{0}\left(E, \operatorname{Cliff}\left(p^{*} E\right)\right) \hat{\otimes}_{M} \mathcal{C}(M)
$$


from Theorem 2.10, there is a graded $*$-homomorphism

$$
\Psi_{p}=\beta_{E} \hat{\otimes}_{M} \operatorname{id}_{M}: \mathcal{A}(M) \rightarrow \mathcal{A}(E)
$$

which on elementary tensors $f \hat{\otimes} s \in \mathcal{S} \hat{\otimes} \mathcal{C}(M)=\mathcal{A}(M)$ is given by

$$
f \hat{\otimes} s \mapsto f\left(X \hat{\otimes} 1+1 \hat{\otimes} C_{E}\right) \hat{\otimes}_{M} s .
$$

Proof. From the discussion above, we have that $\beta_{E} \hat{\otimes}_{M} \operatorname{id}_{M}$ is the composition

$$
\mathcal{S} \hat{\otimes} \mathcal{C}(M) \stackrel{\beta_{E} \hat{\otimes} \operatorname{id}_{M}}{\longrightarrow} M\left(\mathcal{S} C_{0}\left(E, \operatorname{Cliff}\left(p^{*} E\right)\right)\right) \hat{\otimes} \mathcal{C}(M) \rightarrow M\left(\mathcal{S} C_{0}\left(E, \operatorname{Cliff}\left(p^{*} E\right)\right)\right) \hat{\otimes}_{M} \mathcal{C}(M)
$$

Checking on the generator $f(x)=\exp \left(-x^{2}\right)$ of $\mathcal{S}$, we compute that

$$
f\left(X \hat{\otimes} 1+1 \hat{\otimes} C_{E}\right) \hat{\otimes}_{M} s=\exp \left(-x^{2}\right) \hat{\otimes} \exp \left(-\|e\|^{2}\right) \hat{\otimes}_{M} s \in \mathcal{A}(E)
$$

Similarly for $g(x)=x \exp \left(-x^{2}\right)$, we find that

$$
\begin{aligned}
g\left(X \hat{\otimes} 1+1 \hat{\otimes} C_{E}\right) \hat{\otimes}_{M} s & =x \exp \left(-x^{2}\right) \hat{\otimes} \exp \left(-\|e\|^{2}\right) \hat{\otimes}_{M} s \\
& +\exp \left(-x^{2}\right) \hat{\otimes} e \cdot \exp \left(-\|e\|^{2}\right) \hat{\otimes}_{M} s \in \mathcal{A}(E) .
\end{aligned}
$$

It follows that the range of $\Psi_{p}=\beta_{E} \hat{\otimes}_{M} \operatorname{id}_{M}$ is in $\mathcal{A}(E)$ as desired.

Important Note: Compare the similarities of the $C^{\star}$-algebraic formula above to formula (1) for the topological Thom homomorphism $\Phi: K^{0}(M) \rightarrow K^{0}(E)$. Both have the same form: pullback from $M$ and multiply/tensor by the Thom class/operator of the bundle $E$.

We now come to the main theorem of this section:

Thom Isomorphism Theorem 2.14. If $E \rightarrow M$ is a smooth finite rank affine Euclidean bundle, then the *-homomorphism $\Psi_{p}: \mathcal{A}(M) \rightarrow \mathcal{A}(E)$ induces an isomorphism of abelian groups:

$$
\Psi_{*}: K_{*}(\mathcal{A}(M)) \rightarrow K_{*}(\mathcal{A}(E))
$$

The proof will be essentially the same argument as given in another "Thom isomorphism" theorem of the author (Theorem B.22 [37]). But first, we must develop some functorial properties of these Thom maps.

Since the space of compatible connections $\nabla$ on $E \rightarrow M$ is convex, we have the following result.

Proposition 2.15. Let $p: E \rightarrow M$ be a smooth finite rank affine Euclidean bundle. The homotopy class of the *-homomorphism $\Psi_{p}: \mathcal{A}(M) \rightarrow \mathcal{A}(E)$ is independent of the choice of compatible connection $\nabla$ on $E$.

Proposition 2.16. If $p: E=M \times V \rightarrow M$ is a trivial finite rank affine Euclidean bundle (with trivial connection $\nabla_{0}=d$ ) then we have a $\mathbb{Z}_{2}$-graded isomorphism

$$
\mathcal{C}(E) \cong \mathcal{C}(V) \hat{\otimes} \mathcal{C}(M)
$$

such that the Thom map has the form

$$
\Psi_{p} \cong \beta_{V} \hat{\otimes} \operatorname{id}_{\mathcal{C}(M)}: \mathcal{A}(M)=\mathcal{S} \hat{\otimes} \mathcal{C}(M) \rightarrow \mathcal{A}(V) \hat{\otimes} \mathcal{C}(M) \cong \mathcal{A}(E)
$$

where $\beta_{V}: \mathcal{S} \rightarrow \mathcal{A}(V): f \mapsto f\left(X \hat{\otimes} 1+1 \hat{\otimes} C_{V}\right)$ is the Thom map for $V \rightarrow\{0\}$. 
Proof. The trivial connection $\nabla_{0}=d$ gives the manifold $E=M \times V$ the Riemannian metric induced by the isomorphism

$$
T E=T M \times T V \rightarrow M \times V=E .
$$

The pullback vector bundle $p^{*} E \rightarrow E$ has the form

$$
p^{*} E=(M \times V) \times V \rightarrow M \times V=E
$$

and so the Clifford bundle $\operatorname{Cliff}\left(p^{*} E\right)=(M \times V) \times \operatorname{Cliff}(V)$, which gives:

$$
C_{0}\left(E, \operatorname{Cliff}\left(p^{*} E\right)\right)=C_{0}(M \times V,(M \times V) \times \operatorname{Cliff}(V)) \cong C_{0}(V, \operatorname{Cliff}(V)) \hat{\otimes} C_{0}(M) .
$$

By Theorem 2.10, it follows that

$$
\begin{aligned}
\mathcal{C}(E) & \cong C_{0}\left(E, \operatorname{Cliff}\left(p^{*} E\right)\right) \hat{\otimes}_{M} \mathcal{C}(M) \\
& \cong C_{0}(V, \operatorname{Cliff}(V)) \hat{\otimes} C_{0}(M) \hat{\otimes}_{M} \mathcal{C}(M) \\
& \cong \mathcal{C}(V) \hat{\otimes} \mathcal{C}(M) .
\end{aligned}
$$

where we used the isomorphism $C_{0}(M) \hat{\otimes}_{M} \mathcal{C}(M) \cong \mathcal{C}(M)$. The result now easily follows.

For example, if $p: E_{b} \rightarrow E_{a}$ is the orthogonal projection of a finite dimensional Euclidean vector space $E_{b}$ onto a linear subspace $E_{a}$ then $\Psi_{p}=\beta_{b a}$ is the "Bott homomorphism" from Definition 3.1 of Higson-Kasparov-Trout [19].

Lemma 2.17. Let $U$ be an open subset of the Riemannian manifold $M$. The inclusion $i: U \hookrightarrow M$ induces a short exact sequence

$$
0 \longrightarrow \mathcal{A}(U) \stackrel{1 \hat{\otimes} i_{*}}{\longrightarrow} \mathcal{A}(M) \longrightarrow \mathcal{A}(M \backslash U) \longrightarrow 0
$$

of $C^{\star}$-algebras. Thus, $\mathcal{A}(U) \triangleleft \mathcal{A}(M)$ as a (two-sided) $C^{\star}$-ideal.

Proof. Give the open subset $U$ the restriction $g_{U}=i^{*}(g)$ of the Riemannian metric $g$ on $M$. The tangent bundle $T U=\left.T M\right|_{U}=i^{*}(T M)$ is the restriction of $T M$ to $U$. Thus, it follows by universality that

$$
\operatorname{Cliff}(T U)=\operatorname{Cliff}\left(i^{*}(T M)\right)=i^{*} \operatorname{Cliff}(T M)=\left.\operatorname{Cliff}(T M)\right|_{U} .
$$

Since $\mathcal{C}(U)=C_{0}(U, \operatorname{Cliff}(T U))$ we have an exact sequence of $C^{\star}$-algebras

$$
0 \longrightarrow \mathcal{C}(U) \stackrel{i_{*}}{\longrightarrow} \mathcal{C}(M) \longrightarrow \mathcal{C}(M \backslash U) \longrightarrow 0 .
$$

Taking the (maximal) graded tensor product with the nuclear, hence exact $[\mathbf{3 9}]$, $C^{\star}$-algebra $\mathcal{S}=C_{0}(\mathbb{R})$, we obtain an exact sequence of $C^{\star}$-algebras

$$
0 \longrightarrow \mathcal{S} \hat{\otimes} C(U) \stackrel{1 \hat{\otimes} i_{*}}{\longrightarrow} \mathcal{S} \hat{\otimes} C(M) \longrightarrow \mathcal{S} \hat{\otimes} C(M \backslash U) \longrightarrow 0
$$

as desired.

The next result shows that the functor $M \mapsto K_{*}(\mathcal{A}(M))$ has Mayer-Vietoris exact sequences. 
Lemma 2.18. Let $U$ and $V$ be open subsets of $M$. There is an exact sequence

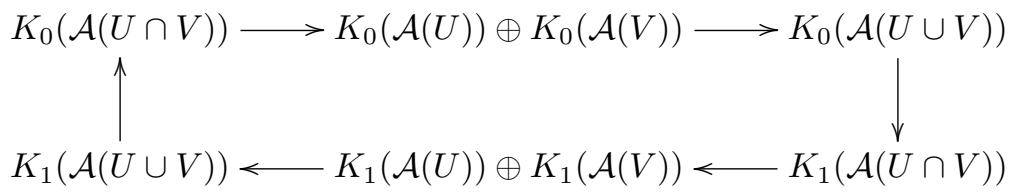

of abelian groups.

Proof. By Lemma 2.17, $\mathcal{A}(U)$ and $\mathcal{A}(V)$ are ideals in $\mathcal{A}(U \cup V)$. By an approximation argument we have the relations:

$$
\left\{\begin{array}{l}
\mathcal{A}(U)+\mathcal{A}(V)=\mathcal{A}(U \cup V) \\
\mathcal{A}(U) \cap \mathcal{A}(V)=\mathcal{A}(U \cap V)
\end{array}\right.
$$

since similar relations hold for $\mathcal{C}(U)$ and $\mathcal{C}(V)$. The result now follows from Exercise $4.10 .21[\mathbf{2 0}]$.

Lemma 2.19. Let $(E, \nabla, M)$ be as above. If $U$ is an open subset of $M$ there is a commutative diagram

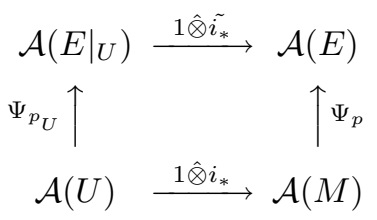

where $p_{U}:\left.E\right|_{U} \rightarrow U$ denotes the restriction of the Euclidean bundle $E$ to $U$.

Proof. Let $i: U \hookrightarrow M$ denote the inclusion of $U$ as an open submanifold of $M$. The restricted bundle $\left.E\right|_{U}=i^{*}(E)$, which as a subset of $E$, is open since $\left.E\right|_{U}=p^{-1}(U)$ as a set. We have a commuting diagram

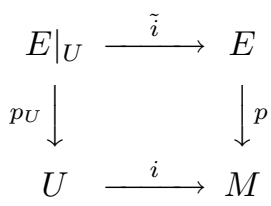

where the horizontal maps are the inclusions as open subsets and the vertical maps are the bundle projections. Give $\left.E\right|_{U}$ the restriction of the Euclidean metric on $E$. Let $\nabla_{U}=i^{*}(\nabla)$ denote the restriction of $\nabla$ to $\left.E\right|_{U}$. The tautological section $\tau_{U}:\left.\left.E\right|_{U} \rightarrow p^{*} E\right|_{U}$ is the restriction $\tau_{U}=\left.\tau\right|_{E_{U}}=\tilde{i}^{*}(\tau)$ of the tautological section $\tau$ of $E$. It follows that the connection 1-form $\theta_{U}=\nabla_{U}^{*} \tau_{U} \in \Omega^{1}\left(\left.E\right|_{U},\left.p^{*} E\right|_{U}\right)$ satisfies

$$
\theta_{U}=\nabla_{U}^{*} \tau_{U}=p_{U}^{*} i^{*}(\nabla) \tilde{i}^{*}(\tau)=\tilde{i}^{*}(\nabla \tau)=\tilde{i}^{*}(\theta)
$$

and so is the restriction of the connection 1-form $\theta \in \Omega^{1}\left(E, p^{*} E\right)$ of $\nabla$ to $\left.E\right|_{U}$. This implies that the horizontal subbundle $H\left(\left.E\right|_{U}\right)$ is

$$
p_{U}^{*} T U \cong H\left(\left.E\right|_{U}\right)=\operatorname{ker} \theta_{U} \cong \tilde{i}^{*}(\operatorname{ker} \theta)=\left.\tilde{i}^{*}(H E) \cong\left(p^{*} T M\right)\right|_{\left.E\right|_{U}} .
$$


Thus, we have coherent splittings

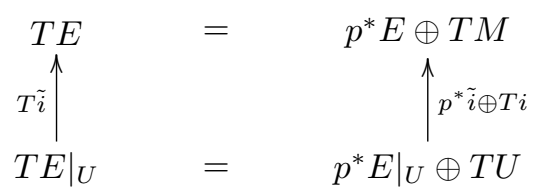

where the vertical maps are open inclusions. This implies that we have an induced commuting diagram

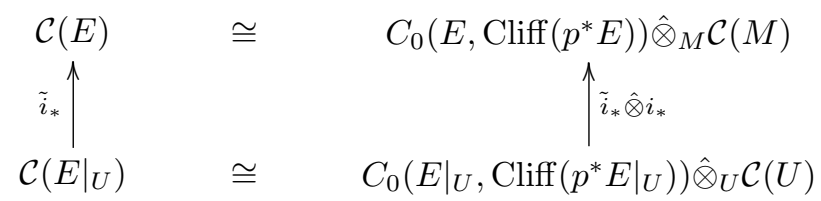

of $C^{\star}$-algebras. Since the Thom operator $C_{E_{U}}:\left.E\right|_{U} \rightarrow \operatorname{Cliff}\left(\left.p^{*} E\right|_{U}\right)$ is the restriction of the Thom operator $C_{E}$ to $\left.E\right|_{U}$, the result easily follows by checking on generators $\exp \left(-x^{2}\right) \hat{\otimes} s_{U}$ and $x \exp \left(-x^{2}\right) \hat{\otimes} s_{U}$ of $\mathcal{S} \hat{\otimes} \mathcal{C}(U)$.

We are now ready to prove the finite rank Thom Isomorphism Theorem 2.14.

Proof of Theorem 2.14. If $M=\{0\}$ is a one-point space, then $E=V$ is a finite dimensional Euclidean space and so, by Lemma 2.4, $\Psi_{p}=\beta_{V}: \mathcal{S}=\mathcal{A}(0) \rightarrow \mathcal{A}(V)$. The induced map on $K$-theory $\Psi_{*}=\beta_{*}: K_{*}(\mathcal{S}) \rightarrow K_{*}(\mathcal{A}(V))$ is an isomorphism by the Bott periodicity theorem 2.6 of Higson-Kasparov-Trout [19].

If $E=M \times V \rightarrow M$ is a trivial bundle, then we may assume that $\nabla=d$ is the trivial connection by Proposition 2.15. Thus, by Lemma 2.4 we have that $\Psi_{p}=\beta_{V} \hat{\otimes} \operatorname{id}_{\mathcal{C}(M)}: \mathcal{A}(M)=\mathcal{S} \hat{\otimes} \mathcal{C}(M) \rightarrow \mathcal{A}(V) \hat{\otimes} \mathcal{C}(M) \cong \mathcal{A}(E)$. The induced map on $K$-theory is an isomorphism, again by the proof of Theorem 2.6 [19], by tensoring with $\operatorname{id}_{\mathcal{C}(M)}$. Indeed, the inverse of $\Psi_{*}$ is induced by

$$
\left\{\phi_{t} \hat{\otimes} \operatorname{id}_{\mathcal{C}(M)}\right\}: \mathcal{A}(V) \hat{\otimes} \mathcal{C}(M) \rightarrow \mathcal{S K}\left(L^{2}(V, \operatorname{Cliff}(V)) \hat{\otimes} \mathcal{C}(M)\right.
$$

where

$$
\left\{\phi_{t}\right\}: \mathcal{A}(V) \rightarrow \mathcal{S K}\left(L^{2}(V, \operatorname{Cliff}(V)): f \hat{\otimes} s \mapsto f\left(X \hat{\otimes} 1+1 \hat{\otimes} t^{-1} D_{V}\right)(1 \hat{\otimes} s)\right.
$$

is the asymptotic morphism of the Dirac operator $D_{V}$ on $L^{2}(V$, Cliff $(V))$ of Definitions $2.7 \& 2.8[\mathbf{1 9}]$.

Let $\left\{U_{n}\right\}_{1}^{\infty}$ be a countable covering by open subsets of $M$ such that $E$ trivializes over each $U_{n}$. We now proceed by induction on $n$. If $n=1$ the result follows by Lemma 2.3, Proposition 2.16 and the discussion above. Suppose we have proved the result for $E$ restricted to $U=U_{1} \cup U_{2} \cup \cdots \cup U_{n}$ (or any open subset of $U$ ). Set $V=U_{n+1}$. By Lemmas 2.17 and 2.19, there is a commuting cubical diagram of 
$C^{\star}$-algebra homomorphisms

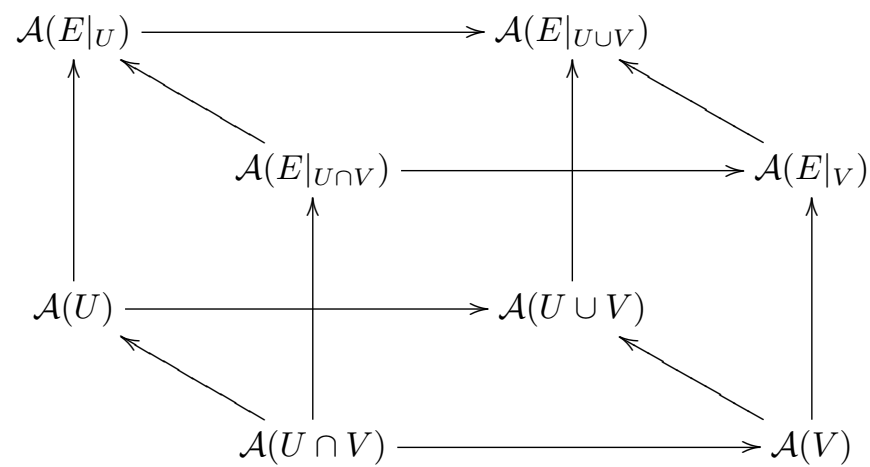

where the vertical arrows are the appropriate Thom maps and the top and bottom squares are inclusions. By Lemma 2.18 we have Mayer-Vietoris sequences

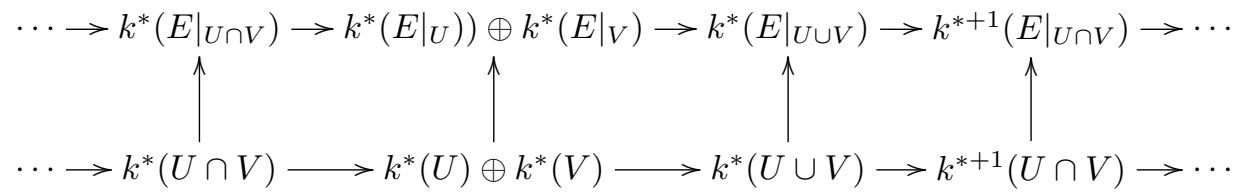

where we have used the simplifying notation $k^{*}(M)=K_{*}(\mathcal{A}(M))$. By induction, the vertical arrows are isomorphisms from $k^{*}(U), k^{*}(V)$, and $k^{*}(U \cap V)$ (since $U \cap V$ is an open subset of $U$.) By the Five Lemma, we conclude that the vertical arrows from $k^{*}(U \cup V)$ are also isomorphisms. This concludes the proof.

Corollary 2.20. If $E$ is a finite even rank oriented Euclidean spinc-bundle (with spin connection $\nabla)$ on an even-dimensional oriented Riemannian spin ${ }^{c}$-manifold $M$, then $\Psi_{p}: \mathcal{A}(M) \rightarrow \mathcal{A}(E)$ induces the topological Thom isomorphism

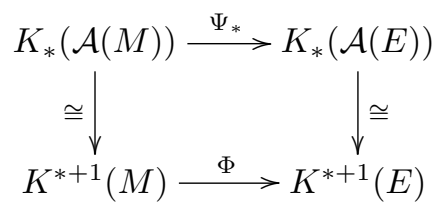

Proof. From the splitting $T E=p^{*} E \oplus p^{*} T M$, we see that $E$ has a canonical structure as an oriented even-dimensional $\operatorname{spin}^{c}-$ manifold. The result now follows by Proposition 2.6, Corollary 2.7, and the previous proof.

\section{The $C^{\star}$-algebra of an Infinite Rank Euclidean Bundle}

In this section, we are going to construct a $C^{\star}$-algebra $\mathcal{A}(\mathfrak{E})$ for an infinite rank Euclidean bundle $\mathfrak{E} \rightarrow M$, analogous to the one in Definition 2.5. It should be stressed that in the infinite dimensional case $\mathcal{A}(\mathfrak{E})$ is not the suspension of a $C^{\star}$ algebra. We choose the notation $\mathcal{A}(\mathfrak{E})$ to be consistent with the notation in [18], which differs from the potentially confusing notation in the original Bott periodicity 
paper [19]. First we need to investigate the transitivity properties of the Thom maps from the previous section.

Fix a smooth finite dimensional Riemannian manifold $M$. Let $p_{2}: E^{2} \rightarrow M$ be a finite rank Euclidean bundle with metric $(\cdot, c d o t)_{2}$. Suppose that $E^{1} \subset E^{2}$ is a vector subbundle of $E^{2}$, i.e., there is an exact sequence of vector bundles

$$
0 \longrightarrow E^{1} \stackrel{i}{\longrightarrow} E^{2} .
$$

Let $p_{1}=\left.p_{2}\right|_{E_{1}}: E^{1} \rightarrow M$ be the subbundle projection.

We can restrict the bundle metric on $E^{2}$ to get a metric $(\cdot, \cdot)_{1}=i^{*}(\cdot, \cdot)_{2}$ on $E^{1}$ so that $i$ is an inclusion of Euclidean bundles. We then have a canonical orthogonal splitting $E^{2}=E^{\perp} \oplus E^{1}$ of Euclidean bundles. Now let $\nabla^{2}$ be a compatible connection on $E^{2}$. We can then define a connection $\nabla^{1}$ on $E^{1}$ by the composition

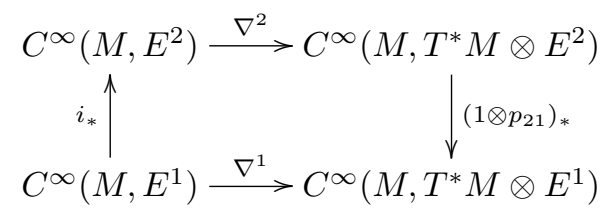

where $p_{21}: E^{2}=E^{\perp} \oplus E^{1} \rightarrow E^{1}$ is the orthogonal bundle projection onto $E^{1}$. The connection $\nabla^{1}$ is the called the projection of the connection $\nabla^{2}$ onto $E^{1}$.

Lemma 3.1. The projected connection $\nabla^{1}$ on the subbundle $E^{1}$ is compatible with the induced metric $(\cdot, \cdot)_{1}$.

Let $E^{21}$ denote the vector bundle $p_{21}: E^{2} \rightarrow E^{1}$. There is then a Euclidean bundle isomorphism $E^{21} \cong p_{1}^{*} E^{\perp}$, and an induced connection $\nabla^{21}=p_{1}^{*} \nabla^{\perp}$, where $\nabla^{\perp}$ is the induced connection on $p_{2}: E^{\perp} \rightarrow M$ via projecting $\nabla^{2}$ as above. (Note that $\nabla^{2}=\nabla^{\perp} \oplus \nabla^{1}$ with respect to the orthogonal splitting $E^{2}=E^{\perp} \oplus E^{1}$.) Hence, we have a commuting diagram of vector bundle projections

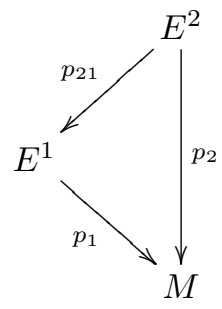

and an associated diagram of Thom $*$-homomorphisms (via Theorem 2.13):

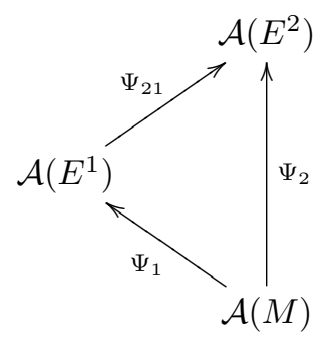


We want to prove that this diagram commutes, i.e., the Thom maps are transitive. The proof is similar to Proposition 3.2 [19], which will be an immediate corollary.

Proposition 3.2. Let $p_{2}: E^{2} \rightarrow M$ be a finite rank Euclidean bundle with connection $\nabla^{2}$. If $p_{1}: E^{1} \rightarrow M$ is a finite rank vector bundle such that there is a vector bundle inclusion $i: E^{1} \hookrightarrow E^{2}$, then diagram (1) above commutes, where $\Psi_{21}: \mathcal{A}\left(E^{1}\right) \rightarrow \mathcal{A}\left(E^{2}\right)$ is the Thom homomorphism associated to the orthogonal projection $p_{21}: E^{2} \rightarrow E^{1}$.

Proof. First we need to check that the Riemannian metric (from Lemma 2.9) on the total space manifold $E^{2}$ induced by the triple $\left(E^{2}, \nabla^{2}, M\right)$ (used to define the $C^{\star}$-algebra $\left.\mathcal{C}\left(E^{2}\right)\right)$ is the same as the Riemannian metric on the manifold $E^{21}=E^{2}$ induced by the triple $\left(E^{21}, \nabla^{12}, E^{1}\right)$. Using the induced metrics and connections from above, we have the following orthogonal splittings of bundles:

$$
\left\{\begin{aligned}
T E^{2} & =p_{2}^{*} E^{2} \oplus p_{2}^{*}(T M) \\
T E^{21} & =p_{21}^{*} E^{21} \oplus p_{21}^{*}\left(T E^{1}\right) \\
T E^{1} & =p_{1}^{*} E^{1} \oplus p_{1}^{*}(T M)
\end{aligned}\right.
$$

By plugging the third into the second and using the fact that $p_{2}=p_{1} \circ p_{21}$, we obtain the first splitting. Hence, these orthogonal splittings are compatible with each other. Therefore, there is a canonical identification $\mathcal{C}\left(E^{2}\right)=\mathcal{C}\left(E^{21}\right)$ of graded $C^{\star}$-algebras.

The orthogonal splitting $E^{2}=E^{\perp} \oplus E^{1}$ induces an orthogonal splitting

$$
p_{2}^{*} E^{2}=p_{2}^{*} E^{\perp} \oplus p_{2}^{*} E^{1}=p_{21}^{*} E^{21} \oplus p_{21}^{*} p_{1}^{*} E^{1} .
$$

This implies that we have a canonical Clifford algebra bundle decomposition

$$
\operatorname{Cliff}\left(p_{2}^{*} E^{2}\right) \cong \operatorname{Cliff}\left(p_{21}^{*} E^{21}\right) \hat{\otimes} p_{21}^{*} \operatorname{Cliff}\left(p_{1}^{*} E^{1}\right) .
$$

This decomposition induces a canonical isomorphism of graded $C^{\star}$-algebras

$$
\begin{aligned}
C_{0}\left(E^{2}, \operatorname{Cliff}\left(p_{2}^{*} E^{2}\right)\right) & \cong C_{0}\left(E^{2}, \operatorname{Cliff}\left(p_{21}^{*} E^{21}\right)\right) \hat{\otimes}_{E^{2}} p_{21}^{*} C_{0}\left(E^{1}, \operatorname{Cliff}\left(p_{1}^{*} E^{1}\right)\right) \\
& =C_{0}\left(E^{21}, \operatorname{Cliff}\left(p_{21}^{*} E^{21}\right)\right) \hat{\otimes}_{E^{2}} p_{21}^{*} C_{0}\left(E^{1}, \operatorname{Cliff}\left(p_{1}^{*} E^{1}\right)\right)
\end{aligned}
$$

where we use the fact that $E^{21}=E^{2}$ as manifolds. Consequently, we have

$$
\mathcal{A}\left(E^{2}\right) \cong \mathcal{S} \hat{\otimes} C_{0}\left(E^{21}, \operatorname{Cliff}\left(p_{21}^{*} E^{21}\right)\right) \hat{\otimes}_{E^{2}} p_{21}^{*} C_{0}\left(E^{1}, \operatorname{Cliff}\left(p_{1}^{*} E^{1}\right)\right) \hat{\otimes}_{M} \mathcal{C}(M) .
$$

Note that since since $p_{21}^{*} C_{0}\left(E^{1}, \operatorname{Cliff}\left(p_{1}^{*} E^{1}\right)\right)=C_{0}\left(E^{2}\right) \hat{\otimes}_{E^{1}} C_{0}\left(E^{1}, \operatorname{Cliff}\left(p_{1}^{*} E^{1}\right)\right)$ this implies that

$$
\begin{aligned}
\mathcal{A}\left(E^{2}\right) & \cong \mathcal{S} \hat{\otimes} C_{0}\left(E^{21}, \operatorname{Cliff}\left(p_{21}^{*} E^{21}\right)\right) \hat{\otimes}_{E^{1}} C_{0}\left(E^{1}, \operatorname{Cliff}\left(p_{1}^{*} E^{1}\right)\right) \hat{\otimes}_{M} \mathcal{C}(M) \\
& \cong \mathcal{S} \hat{\otimes} C_{0}\left(E^{2}, \operatorname{Cliff}\left(p_{21}^{*} E^{21}\right)\right) \hat{\otimes}_{E^{1}} \mathcal{C}\left(E^{1}\right) \cong \mathcal{A}\left(E^{21}\right)
\end{aligned}
$$

which is the decomposition for the Thom map $\Psi_{21}: \mathcal{A}\left(E^{1}\right) \rightarrow \mathcal{A}\left(E^{2}\right)$.

Let $C_{2}=C_{E^{2}}: E^{2} \rightarrow \operatorname{Cliff}\left(p_{2}^{*} E^{2}\right)$ be the Thom operator for $p_{2}: E_{2} \rightarrow M$ and let $C_{1}=C_{E^{1}}: E^{1} \rightarrow \operatorname{Cliff}\left(p_{1}^{*} E^{1}\right)$ be the Thom operator for $p_{1}: E^{1} \rightarrow M$ as in Theorem 2.11. Let $C_{21}: E^{21} \rightarrow \operatorname{Cliff}\left(p_{21}^{*} E^{21}\right)$ be the Thom operator for the bundle $p_{21}: E^{21} \rightarrow E^{1}$. If $e=e^{\perp} \oplus e^{1} \in E^{2}=E^{\perp} \oplus E^{1}$, then one computes:

$$
C_{2}(e)=C_{p_{2}(e)}(e)=C_{p_{21}(e)}\left(e^{\perp}\right) \hat{\otimes} 1+1 \hat{\otimes} C_{p_{1} p_{21}(e)}\left(e^{1}\right)
$$


with respect to the decomposition (4) above. Thus, it implies that we have a tensor product decomposition of essentially self-adjoint unbounded multipliers

$$
C_{2}=C_{21} \hat{\otimes} 1+1 \hat{\otimes} p_{21}^{*} C_{1}
$$

induced by the $C^{\star}$-algebra decomposition above.

It suffices to compute the composition $\Psi_{21} \circ \Psi_{1}$ on generators of $\mathcal{A}(M)=\mathcal{S} \hat{\otimes} \mathcal{C}(M)$ of the form $\exp \left(-x^{2}\right) \hat{\otimes} s$ and $x \exp \left(-x^{2}\right) \hat{\otimes} s$ where $s \in \mathcal{C}(M)$. We obtain (using isomorphisms (5) and (6)) the elements, respectively ${ }^{4}$,

$$
\exp \left(-x^{2}\right) \hat{\otimes} \exp \left(-C_{21}^{2}\right) \hat{\otimes} p_{21}^{*} \exp \left(-C_{1}^{2}\right) \hat{\otimes} s
$$

and

$$
\begin{array}{r}
x \exp \left(-x^{2}\right) \hat{\otimes} \exp \left(-C_{21}^{2}\right) \hat{\otimes} p_{21}^{*} \exp \left(-C_{1}^{2}\right) \hat{\otimes} s \\
+\exp \left(-x^{2}\right) \hat{\otimes} C_{21} \exp \left(-C_{21}^{2}\right) \hat{\otimes} p_{21}^{*} \exp \left(-C_{1}^{2}\right) \hat{\otimes} s \\
+\exp \left(-x^{2}\right) \hat{\otimes} \exp \left(-C_{21}^{2}\right) \hat{\otimes} p_{21}^{*} C_{1} \exp \left(-C_{1}^{2}\right) \hat{\otimes} s
\end{array}
$$

However, by Corollary A.4, we have equalities

$$
\exp \left(-C_{2}^{2}\right)=\exp \left(-C_{21}\right)^{2} \hat{\otimes} p_{21}^{*} \exp \left(-C_{1}^{2}\right)
$$

and also

$$
C_{2} \exp \left(-C_{2}^{2}\right)=C_{21} \exp \left(-C_{21}^{2}\right) \hat{\otimes} p_{21}^{*} \exp \left(-C_{1}^{2}\right)+\exp \left(-C_{21}^{2}\right) \hat{\otimes} p_{21}^{*}\left(C_{1} \exp \left(-C_{1}^{2}\right)\right) .
$$

But under the map $\Psi_{2}$ the generators $\exp \left(-x^{2}\right) \hat{\otimes} s$ and $x \exp \left(-x^{2}\right) \hat{\otimes} s$ are mapped, respectively, to the elements

$$
\exp \left(-x^{2}\right) \hat{\otimes} \exp \left(-C_{2}^{2}\right) \hat{\otimes} s
$$

and

$$
x \exp \left(-x^{2}\right) \hat{\otimes} \exp \left(-C_{2}^{2}\right) \hat{\otimes} s+\exp \left(-x^{2}\right) \hat{\otimes} C_{2} \exp \left(-C_{2}^{2}\right) \hat{\otimes} s .
$$

Therefore, the composition $\Psi_{21} \circ \Psi_{1}$ agrees with $\Psi_{2}$ on generators since $p_{2}=p_{1} \circ p_{21}$, and hence the two maps are equal.

Let $\mathcal{E}$ be a complete Euclidean space of infinite dimension. Denote by $O(\mathcal{E})$ the orthogonal group of all invertible bounded linear maps $T: \mathcal{E} \rightarrow \mathcal{E}$ which preserve the inner product:

$$
\langle T(v), T(w)\rangle=\langle v, w\rangle, \quad \forall v, w \in \mathcal{E} .
$$

Let $\mathfrak{E}$ be an infinite dimensional manifold $[\mathbf{2 , 2 5}]$ modelled on $\mathcal{E}$.

Definition 3.3. A smooth map $p: \mathfrak{E} \rightarrow M$ is a Euclidean bundle modelled on $\mathcal{E}$ if it defines a smooth locally trivial fiber bundle $\mathfrak{E}$ with fiber $\mathcal{E}$ and structure group $O(\mathcal{E})$ in the norm (or strong operator) topology [25, 30].

It follows that $\mathfrak{E}$ is equipped with a metric $\langle\cdot, \cdot\rangle_{x}$ on each fiber $\mathfrak{E}_{x}$, which varies smoothly in $x \in M$. That is, the map $(e, f) \mapsto\langle e, f\rangle_{p(e)}$ is smooth on the fiber product $\mathfrak{E} \times{ }_{M} \mathfrak{E}=\{(e, f) \mid p(e)=p(f)\}$.

${ }^{4}$ We drop the subscripts on tensor products for clarity. 
Definition 3.4. A subset $S^{a} \subset \mathfrak{E}$ will be called a subbundle if there is a real vector bundle $p_{a}: E^{a} \rightarrow M$ and an exact sequence

$$
0 \longrightarrow E^{a} \stackrel{i}{\longrightarrow} \mathfrak{E},
$$

of vector bundles such that $i\left(E^{a}\right)=S^{a}$. That is, $i: E^{a} \rightarrow \mathfrak{E}$ is a vector bundle morphism such that for each $x \in M$ the continuous linear map $i_{x}: E_{x}^{a} \rightarrow \mathfrak{E}_{x}$ on fibers is split injective (i.e., with split range). This gives $S^{a}$ the (unique) structure of a vector bundle, and we will identify $E^{a} \cong S^{a} \subseteq \mathfrak{E}$. If $\operatorname{rank}\left(E^{a}\right)=n<\infty$ then we will call $E^{a} \subset \mathfrak{E}$ a finite rank subbundle.

The following is adapted from Proposition 3.4.18 [2].

Proposition 3.5. Let $p: \mathfrak{E} \rightarrow M$ be a Euclidean bundle modelled on $\mathcal{E}$ and let $q: E \rightarrow M$ be a finite rank vector bundle. If $f: E \rightarrow \mathfrak{E}$ is a vector bundle map let $f_{x}: E_{x} \rightarrow \mathfrak{E}_{x}$ be the linear restriction to the fibers over $x \in M$ and let range $(f)=\bigcup_{x \in M}$ range $\left(f_{x}\right)$. Then range $(f) \subset \mathfrak{E}$ is a finite rank subbundle if and only if $x \mapsto \operatorname{rank}\left(f_{x}\right)$ is locally constant on $M$.

Theorem 3.6. Suppose $\mathcal{E}$ has countably infinite dimension. If $p: \mathfrak{E} \rightarrow M$ is a Euclidean bundle modelled on $\mathcal{E}$, there is an increasing sequence

$$
M=E^{0} \subset E^{1} \subset E^{2} \subset \cdots \subset E^{n} \subset \cdots \subset \mathfrak{E}
$$

of finite rank subbundles such that $\operatorname{rank}\left(E^{n}\right)=n$ and $\mathfrak{E}=\bigcup_{0}^{\infty} E^{n}$.

Proof. The locally trivial fiber bundles with structure group $O(\mathcal{E})$ are classified by the cocycles in the sheaf cohomology $H^{1}(M, O(\mathcal{E})$ ) (which is not a group since $O(\mathcal{E})$ is not abelian) [34]. However, by Kuiper's Theorem [24], the group $O(\mathcal{E})$ is contractible in the norm (or strong operator) topology. Hence, there is only the trivial cocycle corresponding to $\mathfrak{F}=M \times \mathcal{E} \rightarrow M$, the trivial Euclidean bundle over $M$ modelled on $\mathcal{E}$. Thus, there is a vector bundle isomorphism $\Phi: \mathfrak{F} \rightarrow \mathfrak{E}$. Since $\mathcal{E}$ has countably infinite dimension, there is an increasing sequence

$$
\{0\}=V^{0} \subset V^{1} \subset V^{2} \subset \cdots \subset \mathcal{E}
$$

of subspaces of $\mathcal{E}$ such that $\operatorname{dim}\left(V^{n}\right)=n$. Let $F^{n}=M \times V^{n} \subset \mathfrak{F}$ be the associated finite rank subbundle of $\mathfrak{F}$ for all $n \geqslant 0$. It follows that if we define $E^{n}=\Phi\left(F^{n}\right) \subset \mathfrak{E}$, then the sequence $\left\{E^{n}\right\}_{0}^{\infty}$ is our desired increasing sequence of finite rank subbundles.

Important Note: Although every Euclidean bundle $\mathfrak{E}$ with infinite dimensional fiber is trivializable, the finite dimensional subbundles are not necessarily trivializable. Moreover, if a group $G$ is acting on $E$ and $M$ in a compatible way, as we will consider in the next section, then $\mathfrak{E}$ itself may not be equivariantly trivializable.

Definition 3.7. Let $E^{a}$ and $E^{b}$ be finite rank subbundles of $\mathfrak{E}$. We will define $E^{a} \preceq E^{b}$ if $E^{a} \subseteq E^{b}$ as subsets of $\mathfrak{E}$ and there is an exact sequence $0 \rightarrow E^{a} \rightarrow E^{b}$ 
of vector bundles such that the following diagram commutes:

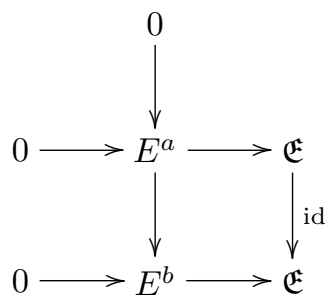

That is, $E^{a}$ is a subbundle of $E^{b}$ in a way that is compatible with their subbundle structures from $\mathfrak{E}$.

Definition 3.8. Let $p: \mathfrak{E} \rightarrow M$ be a Euclidean bundle bundle modelled on $\mathcal{E}$. Denote by $\mathcal{F B}(\mathfrak{E})$ the collection of all smooth finite rank subbundles $p_{a}: E^{a} \rightarrow M$. Note that $\mathcal{F} \mathcal{B}(\mathfrak{E})$ is a directed system under inclusion $\preceq$ of subbundles.

Given a finite rank subbundle $E^{a} \subset \mathfrak{E}$ there is an induced Euclidean metric $\langle\cdot, \cdot\rangle^{a}$, which on the fibers $E_{x}^{a}$ is given by restricting the metric $\langle\cdot, \cdot\rangle_{x}$ of the fiber $\mathfrak{E}_{x}$ to the subspace $E_{x}^{a} \subset \mathfrak{E}_{x}$. We also need a compatible connection $\nabla^{a}$ on $E^{a}$, and this can be done by equipping $\mathfrak{E} \rightarrow M$ with a compatible connection.

Lemma 3.9. Let $p: \mathfrak{E} \rightarrow M$ be a Euclidean bundle modelled on $\mathcal{E}$. There exists a connection $\nabla: C^{\infty}(M, \mathfrak{E}) \rightarrow C^{\infty}\left(M, T^{*} M \otimes \mathfrak{E}\right)$ which is compatible with the Euclidean metric $\langle\cdot, \cdot\rangle$ on $\mathfrak{E}$.

Proof. Standard partition of unity argument.

If $E^{a} \subset \mathfrak{E}$ is a finite rank subbundle, then there is an orthogonal decomposition of Euclidean bundles $\mathfrak{E}=E^{\perp} \oplus E^{a}$. Let $p_{a}^{\perp}: \mathfrak{E} \rightarrow E^{a}$ denote the orthogonal projection onto $E^{a}$. We can then induce (project) a connection $\nabla^{a}$ on $E^{a}$ by the diagram

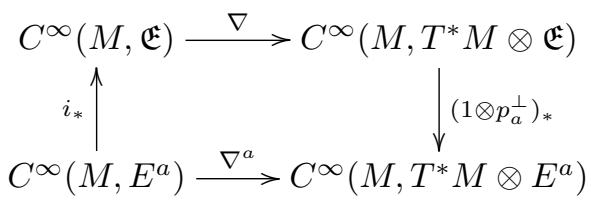

One can then easily check that $\nabla^{a}$ is compatible with the induced metric $\langle\cdot, \cdot\rangle^{a}$.

Given any two finite rank subbundles $E^{a} \preceq E^{b}$ of $\mathfrak{E}$, we then have a canonical orthogonal decomposition $E^{b} \cong E_{a b}^{\perp} \oplus E^{a}$ Thus, we can define a bundle projection $p_{a b}: E^{b} \rightarrow E^{a}$. Let $E^{b a}$ denote the vector bundle $p_{a b}: E^{b} \rightarrow E^{a}$. By the discussion prior to Proposition 3.2, this has a Euclidean bundle structure given by $E^{b a} \cong p_{a}^{*} E_{a b}^{\perp}$ and compatible connection $\nabla^{a b}=p_{a}^{*} \nabla^{\perp}$ where $\nabla^{b}=\nabla^{\perp} \oplus \nabla^{a}$. By Theorem 2.13 there is a Thom $*$-homomorphism

$$
\Psi_{a b}: \mathcal{A}\left(E^{a}\right) \rightarrow \mathcal{A}\left(E^{b}\right)
$$

which will be the connecting map in our construction of the $\operatorname{direct} \operatorname{limit} C^{\star}$-algebra associated to $\mathfrak{E}$. 
Lemma 3.10. Let $p: \mathfrak{E} \rightarrow M$ be an affine Euclidean bundle modelled on $\mathcal{E}$. If $E^{a} \preceq E^{b} \preceq E^{c}$ are finite rank affine subbundles of $\mathfrak{E}$, then there is a commutative diagram of Thom *-homomorphisms:

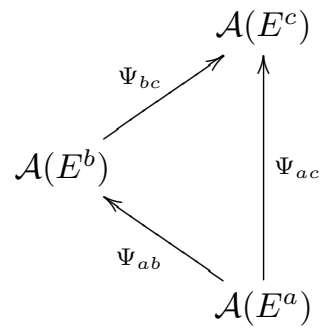

Proof. Note that since $E^{a}, E^{b}$ and $E^{c}$ are fibered as Euclidean bundles with compatible connections over the Riemannian manifold $M$, they have canonical Riemannian structures, as manifolds, from Lemma 2.9. We also have a commuting diagram of bundle projections

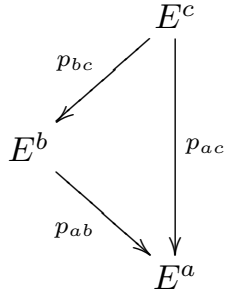

Note that the Riemannian structure that $E^{c}$ has as a bundle over $E^{a}$ and $E^{b}$ is compatible with the structure coming from $E^{c} \rightarrow M$. Similarly for $E^{b}$. The result now follows from Proposition 3.2

Definition 3.11. Let $p: \mathfrak{E} \rightarrow M$ be a smooth affine Euclidean bundle modelled on $\mathcal{E}$. We define $\mathcal{A}(\mathfrak{E})$ to be the direct limit $C^{\star}$-algebra

$$
\mathcal{A}(\mathfrak{E})=\underset{a}{\lim } \mathcal{A}\left(E^{a}\right),
$$

where the direct limit is taken over the directed system $\mathcal{F} \mathcal{B}(\mathfrak{E})$ of all finite rank subbundles $E^{a} \subset \mathfrak{E}$, using the $*$-homomorphisms $\Psi_{a b}: \mathcal{A}\left(E^{a}\right) \rightarrow \mathcal{A}\left(E^{b}\right)$ above.

Note that since $\mathfrak{E}$ is infinite dimensional, $\mathcal{A}(\mathfrak{E})$ is not the suspension of a $C^{\star}$ algebra. However, $\mathcal{A}(\mathfrak{E})$ is a graded, separable, nuclear $C^{\star}$-algebra.

By transitivity again, we have that the $*$-homomorphisms

$$
\Psi_{a}: \mathcal{A}(M) \rightarrow \mathcal{A}\left(E^{a}\right)
$$

are compatible with the direct limit, so there is a canonical Thom map

$$
\Psi_{p}: \mathcal{A}(M) \rightarrow \underset{a}{\lim } \mathcal{A}\left(E^{a}\right)=\mathcal{A}(\mathfrak{E}) .
$$

Note that this is equivalent to identifying $M$ as the zero finite rank subbundle (section) of $\mathfrak{E} \rightarrow M$. 
Lemma 3.12. If $M=\{0\}$ is a point, so that $\mathfrak{E}=\mathcal{E} \rightarrow\{0\}$, then

$$
\Psi=\beta: \mathcal{A}(0) \rightarrow \mathcal{A}(\mathcal{E})
$$

is the Bott homomorphism of Higson-Kasparov-Trout [19]

By the finite rank Thom isomorphism theorem 2.14, the connecting maps $\Psi_{a b}$ : $\mathcal{A}\left(E^{a}\right) \rightarrow \mathcal{A}\left(E^{b}\right)$ induce isomorphisms in $K$-theory:

$$
\Psi_{a b *}: K_{*}\left(\mathcal{A}\left(E^{a}\right)\right) \rightarrow K_{*}\left(\mathcal{A}\left(E^{b}\right)\right) .
$$

Using the continuity of operator $K$-theory with respect to direct limits [40], we obtain:

$$
K_{*}(\mathcal{A}(\mathfrak{E}))=K_{*}\left(\underset{a}{\lim _{\longrightarrow}} \mathcal{A}\left(E^{a}\right)\right) \cong \underset{a}{\lim _{\vec{a}}} K_{*}\left(\mathcal{A}\left(E^{a}\right)\right) \cong K_{*}(\mathcal{A}(M)) .
$$

Therefore, we have proved the non-equivariant

Thom Isomorphism Theorem 3.13. Let $\mathfrak{E}$ be an affine Euclidean bundle on $M$ modelled on $\mathcal{E}$. The inclusion $M \subset \mathfrak{E}$ as the zero subbundle induces an isomorphism in $K$-theory:

$$
\Psi_{*}: K_{*}(\mathcal{A}(M)) \rightarrow K_{*}(\mathcal{A}(\mathfrak{E}))
$$

\section{The Equivariant Thom Isomorphism}

In order to formulate the equivariant version of our theorem, we need to choose an appropriate class of group actions. The most popular class of group actions on manifolds are proper actions because of their application to $K$-theory [30], the index theory of elliptic operators [23, 27], and Baum-Connes Conjectures [6]. Since our theorem must reduce to the Bott periodicity theorem $[\mathbf{1 9}]$ when $M=\{\bullet\}$ is a point, we must allow for the trivial action of a non-compact group on a point. But, non-compact groups do not act properly on a point.

Let $G$ be a smooth, second countable, locally compact, Hausdorff topological group (e.g., a countable discrete group.) A locally compact space $M$ is a $G$-space if it has a continuous (or smooth if $M$ is smooth) $G$-action

$$
G \times M \rightarrow M .
$$

If $H$ is a closed subgroup of $G$, and $x \in M$ then an $H$-slice through $x$ is an $H$ invariant open neighborhood $U$ of $x$ such that the map $G \times U \rightarrow M$ given by $(g, u) \mapsto g u$ descends to a $G$-equivariant homeomorphism

$$
G \times{ }_{H} U=(G \times U) / H \rightarrow G U
$$

and such that $G U$ is an open neighborhood of $x \in M$. If $M$ is a smooth manifold on which $G$ acts smoothly via diffeomorphisms, then we require that this be a $G$-equivariant diffeomorphism.

Definition 4.1. Let $(M, d)$ be a locally compact metric $G$-space. The action of $G$ on $M$ is isoreductive if:

(i) $G$ acts by isometries of $(M, d)$; 
(ii) for every $x \in M$ there is a closed subgroup $H$ of $G$ and an $H$-slice $U$ through $x$ which is $H$-equivariantly contractible.

Note that if $M=\{\bullet\}$ is a point, the trivial $G$-action is vacuously isoreductive since we can choose $H=G$ and $G \times_{G}\{\bullet\}=\{\bullet\}$. If $G$ is a countable discrete group and $M$ is a discrete $G$-space, then $M$ is isoreductive with respect to the trivial metric $d(x, y)=1(x \neq y)$ since we can choose $U=\{x\}=B(x, 1), H=G_{x}$ (the isotropy subgroup of $x$ ) and $G \times \times_{G_{x}}\{x\} \cong G \cdot x$ is the orbit of $x$.

Recall that an action of $G$ on $M$ is called proper $[\mathbf{1}, \mathbf{3 0}]$ if the structural map

$$
\begin{aligned}
G \times M & \rightarrow M \times M \\
(g, m) & \mapsto(g m, m)
\end{aligned}
$$

is a proper map, i.e., the inverse image of a compact set is compact.

For the following, see the discussion in Example (1.4) [6].

Lemma 4.2. If $G$ is a countable discrete group, every smooth isometric proper action of $G$ on a Riemannian manifold is isoreductive.

Indeed, using a $G$-partition of unity [30] we can average any metric on $M$ to obtain a $G$-invariant metric. Also, for any $x \in M$ we can choose $H=G_{x}$ the (finite) isotropy subgroup of $x$ and $G_{x}$-slice consisting of an open ball of small enough radius.

Recall that a $C^{\star}$-algebra $A$ is called a $G-C^{\star}$-algebra if $G$ acts continuously on $A$ by $C^{\star}$-algebra automorphisms (i.e., for every $a \in A$ the map $G \rightarrow A$ given by $g \mapsto g a$ is continuous.)

Lemma 4.3. An isometric action of $G$ on $M$ induces a continuous action of $G$ on $\mathcal{A}(M)$ as $\mathbb{Z}_{2}$-graded $C^{\star}$-algebra automorphisms.

Proof. Given the isometry $g: M \rightarrow M$ there is an induced Euclidean bundle isomorphism $T g: T M \rightarrow T M$ of the tangent bundle $T M$. This induces an isomorphism $\widehat{T g}: \operatorname{Cliff}(T M) \rightarrow \operatorname{Cliff}(T M)$ of the $\mathbb{Z}_{2}$-graded Clifford bundle. Hence, we obtain an induced automorphism $g_{\tau}: \mathcal{C}(M) \rightarrow \mathcal{C}(M)$ of $\mathbb{Z}_{2}$-graded $C^{\star}$-algebras given by $\left(g_{\tau} s\right)(x)=\widehat{T g}\left(s\left(g^{-1} x\right)\right)$ for all $x \in M$ and $s \in \mathcal{C}(M)$. Tensoring with id $\sin _{\mathcal{S}}$ gives the automorphism $g_{*}=\operatorname{id}_{\mathcal{S}} \hat{\otimes} g_{\tau}: \mathcal{S C}(M)=\mathcal{A}(M) \rightarrow \mathcal{A}(M)$.

Definition 4.4. If $A$ is a $G$ - $C^{\star}$-algebra, we define the equivariant $K$-theory $K_{*}^{G}(A)$ to the be the $K$-theory of the full crossed product $C^{\star}$-algebra $A \rtimes G$ :

$$
K_{j}^{G}(A)=K_{j}(A \rtimes G), \quad j=0,1 .
$$

For compact groups, the above definition is actually the Green-Julg-Rosenberg Theorem 11.7.1 [8]. See Pedersen [28] for a discussion of full crossed product $C^{\star}$ algebras. It follows that if $\phi: A \rightarrow B$ is an equivariant $*$-homomorphism then there is an induced $C^{\star}$-algebra $*$-homomorphism $\phi^{G}: A \rtimes G \rightarrow B \rtimes G$. Hence, there is an induced homomorphism $\phi_{*}^{G}: K_{*}^{G}(A) \rightarrow K_{*}^{G}(B)$ of equivariant $K$-theory groups. We chose the full crossed product because of this universal property and its compatibility with equivariant $E$-theory $[\mathbf{1 7}]$. 
Note that if the action of $G$ on $M$ is proper then $K_{G}^{*}(M)$, the equivariant topological $K$-theory of $M$ (as defined by N.C. Phillips [30]) exists. This $K$-theory satisfies a generalized Green-Julg-Rosenberg theorem:

$$
K_{G}^{*}(M) \cong K_{*}\left(C_{0}(M) \rtimes_{\alpha} G\right)
$$

where $C_{0}(M) \rtimes_{\alpha} G$ is any (e.g., full or reduced) crossed product of $C_{0}(M)$ by $G$.

Proposition 4.5. If $M$ is an oriented even-dimensional spin ${ }^{c}$-manifold and $G$ acts on $M$ properly such that the spin ${ }^{c}$-structure is $G$-invariant, then there are canonical isomorphisms

$$
K_{*}^{G}(\mathcal{A}(M)) \cong K_{G}^{*+1}(M)
$$

in equivariant $K$-theory.

¿From now on, we assume that $M$ is a smooth finite-dimensional $G$-manifold equipped with a fixed $G$-invariant Riemannian metric and the action of $G$ is isoreductive with respect to this metric.

Definition 4.6. Let $p_{a}: E^{a} \rightarrow M$ and $p_{b}: E^{b} \rightarrow M$ be smooth Euclidean bundles on $M$. If $g: M \rightarrow M$ is an isometry, then a bundle map $\tilde{g}: E^{a} \rightarrow E^{b}$ is called a Euclidean bundle isomorphism over $g$ if the following diagram commutes

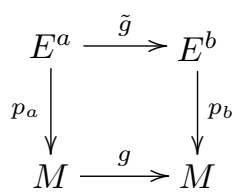

and $\tilde{g}_{x}: E_{x}^{a} \rightarrow E_{g(x)}^{b}$ is an isomorphism of Euclidean spaces for each $x \in M$.

Let $\mathcal{E}$ be a Euclidean space of countably infinite dimension.

Definition 4.7. A Euclidean $G$-bundle $\mathfrak{E}$ on $M$ with fiber $\mathcal{E}$ is a smooth Euclidean bundle $p: \mathfrak{E} \rightarrow M$ modelled on $\mathcal{E}$ equipped with a smooth action $G \times \mathfrak{E} \rightarrow \mathfrak{E}$ by Euclidean bundle automorphisms such that the projection $p: \mathfrak{E} \rightarrow M$ is $G$ equivariant. That is, for each $g \in G$ there is a Euclidean bundle automorphism $\tilde{g}: \mathfrak{E} \rightarrow \mathfrak{E}$ over $g: M \rightarrow M$ such that $\tilde{g}_{1} \circ \tilde{g}_{2}=\tilde{g}$ where $g=g_{1} g_{2} \in G$.

Lemma 4.8. Let $p: \mathfrak{E} \rightarrow M$ be a Euclidean $G$-bundle with fiber $\mathcal{E}$. Then for any smooth finite rank subbundle $E^{a} \subset \mathfrak{E}$ and $g \in G$, the image $g \cdot E^{a}$ has a canonical structure as a finite rank subbundle and

$$
\tilde{g}_{!}=\left.\tilde{g}\right|_{E^{a}}: E^{a} \rightarrow g \cdot E^{a}
$$

is a finite rank Euclidean bundle isomorphism over $g: M \rightarrow M$.

Proof. By Definition 3.4, there is a subset $S^{a} \subset \mathfrak{E}$, a finite rank Euclidean bundle $p_{a}: E^{a} \rightarrow M$ and an exact sequence of Euclidean bundles

$$
0 \longrightarrow E^{a} \stackrel{i_{a}}{\longrightarrow} \mathfrak{E}
$$


such that $i\left(E^{a}\right)=S^{a}$. Now, define the subbundle $g \cdot E^{a} \subset \mathfrak{E}$ as follows. As a subset of $\mathfrak{E}$ we have $g \cdot E^{a}=g \cdot i_{a}\left(E^{a}\right)=g \cdot S^{a}=\left\{g \cdot e: e \in S^{a}\right\}$. As a finite rank Euclidean bundle we use $p_{a}: E^{a} \rightarrow M$ but with associated exact sequence

$$
0 \longrightarrow E^{a} \stackrel{\tilde{g} \circ i_{a}}{\longrightarrow} \mathfrak{E}
$$

given by the composition

$$
0 \longrightarrow E^{a} \stackrel{i_{a}}{\longrightarrow} \mathfrak{E} \stackrel{\tilde{g}}{\longrightarrow} \mathfrak{E}
$$

It follows that there is a commutative diagram

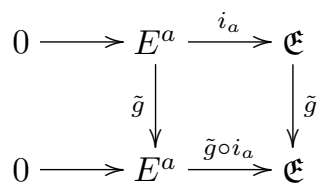

where $\tilde{g}_{!}: E^{a} \rightarrow E^{a}$ is the Euclidean bundle automorphism $i_{a}^{-1} \circ \tilde{g} \circ i_{a}: E^{a} \rightarrow E^{a}$.

Remark 4.9. We should make the important observation that the action of $G$ on $\mathfrak{E}$ may not preserve any finite rank subbundle except, of course, the zero subbundle $M \subset \mathfrak{E}$. Also, if the action of $G$ on $M$ has no fixed points, then the fibers $\mathfrak{E}_{x} \cong \mathcal{E}$ may carry no action of $G$.

Definition 4.10. Let $\left(E^{a}, \nabla^{a}\right)$ and $\left(E^{b}, \nabla^{b}\right)$ be Euclidean bundles on $M$ with compatible connections. Let $g: M \rightarrow M$ be an isometry of $M$. If $\tilde{g}: E^{a} \rightarrow E^{b}$ is a Euclidean bundle isomorphism over $g$, then we call $\tilde{g}$ an affine Euclidean isomorphism (over $g$ ) if the following diagram commutes:

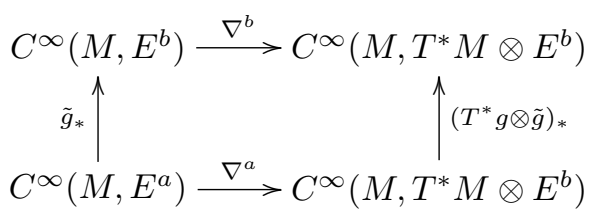

where $T^{*} g: T^{*} M \rightarrow T^{*} M$ is the induced Euclidean bundle isomorphism of the cotangent bundle $T^{*} M \cong T M$. We denote this by $(\tilde{g}, g):\left(E^{a}, \nabla^{a}\right) \rightarrow\left(E^{b}, \nabla^{b}\right)$.

Lemma 4.11. Let $(\tilde{g}, g):\left(E^{a}, \nabla^{a}\right) \rightarrow\left(E^{b}, \nabla^{b}\right)$ be an affine Euclidean isomorphism of finite rank affine Euclidean bundles on $M$. There is an induced isomorphism of $\mathbb{Z}_{2}$-graded $C^{\star}$-algebra $(\tilde{g}, g)_{*}: \mathcal{A}\left(E^{a}\right) \rightarrow \mathcal{A}\left(E^{b}\right)$ such that the following diagram commutes

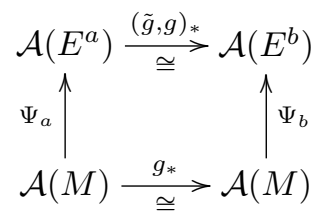

where $g_{*}$ is the isomorphism from Lemma 4.3 and $\Psi_{a}$ and $\Psi_{b}$ are the Thom maps from Theorem 2.13. 
Proof. Using the connections and the fact that $(\tilde{g}, g)$ transforms the connection $\nabla^{a}$ into the connection $\nabla^{b}$, there is a commutative diagram

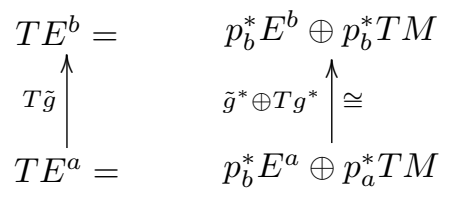

where the vertical maps appearing on the RHS are the Euclidean bundle isomorphisms induced by the pair $(\tilde{g}, g)$. Thus, with the metrics induced by the splitting, the induced map $T \tilde{g}: T E^{a} \rightarrow T E^{b}$ is a Euclidean bundle isomorphism, which implies that $\tilde{g}: E^{a} \rightarrow E^{b}$ induces an isometry between the Riemannian manifolds $E^{a}$ and $E^{b}$.

There is also an induced commutative diagram

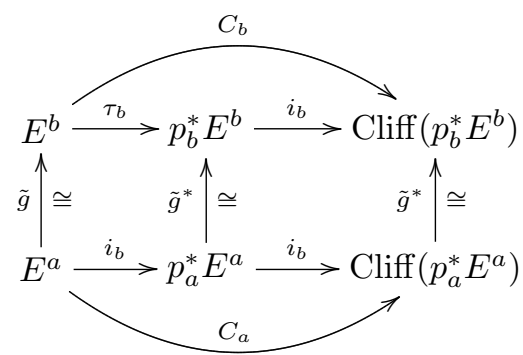

where the vertical maps are the appropriate bundle isomorphisms induced by the map $\tilde{g}$. Thus, the isomorphism $\tilde{g}$ transforms the Thom operator $C_{a}$ of $E^{a}$ into the Thom operator $C_{b}$ of $E^{b}$ in a manner compatible with the isometric action of $g: M \rightarrow M$. The result now easily follows using Theorems 2.10 and 2.13 and the equivariant properties of the functional calculus.

Definition 4.12. Let $p: \mathfrak{E} \rightarrow M$ be a Euclidean $G$-bundle. A connection $\nabla$ on $\mathfrak{E}$ is called $G$-invariant if for each $g \in G$ the bundle map $\tilde{g}: \mathfrak{E} \rightarrow \mathfrak{E}$ determines an affine Euclidean isomorphism $(\tilde{g}, g):(\mathfrak{E}, \nabla) \rightarrow(\mathfrak{E}, \nabla)$. That is,

$$
\left(T^{*} g \otimes \tilde{g}\right)_{*} \circ \nabla=\nabla \circ \tilde{g}_{*}
$$

for all $g \in G$. If $\mathfrak{E}$ is equipped with a $G$-invariant connection, then we say that $\mathfrak{E}$ is an affine Euclidean $G$-bundle.

Averaging any connection on $\mathfrak{E}$ with respect to a $G$-partition of unity yields

Lemma 4.13. If the action of $G$ on $M$ is proper, there exists a $G$-invariant connection $\nabla$ on any Euclidean $G$-bundle $p: \mathfrak{E} \rightarrow M$.

Lemma 4.14. Let $p: \mathfrak{E} \rightarrow M$ be an affine Euclidean $G$-bundle with $G$-invariant connection $\nabla$. Let $E^{a} \subset \mathfrak{E}$ be a finite rank subbundle. The map $\tilde{g}_{!}: E^{a} \rightarrow g \cdot E^{a}$ (from Lemma 4.8) determines an affine Euclidean bundle isomorphism

$$
\left(\tilde{g}_{!}, g\right):\left(E^{a}, \nabla^{a}\right) \rightarrow\left(g \cdot E^{a}, g \cdot \nabla^{a}\right)
$$

where $\nabla^{a}$ denotes the connection induced on $E^{a}$ by $\nabla$. 
Corollary 4.15. With the above hypotheses on $(\mathfrak{E}, \nabla, M, G)$, we have that for each finite rank subbundle $E^{a} \subset \mathfrak{E}$ and each $g \in G$ there is a commutative diagram

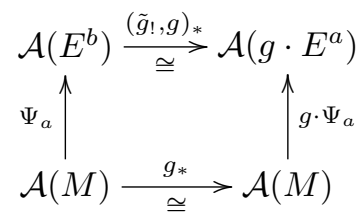

where $g \cdot \Psi_{a}: \mathcal{A}(M) \rightarrow \mathcal{A}\left(g \cdot E^{a}\right)$ is the Thom map for the finite rank subbundle $g \cdot E^{a} \subset \mathfrak{E}$.

Lemma 4.16. With the above hypotheses on $(\mathfrak{E}, \nabla, M, G)$, there is an induced continuous action of $G$ on the direct limit $C^{\star}$-algebra

$$
\mathcal{A}(\mathfrak{E})=\underset{a}{\lim _{a}} \mathcal{A}\left(E^{a}\right)
$$

as $\mathbb{Z}_{2}$-graded $C^{\star}$-algebra automorphisms.

Moreover, the $G$-equivariant inclusion $M \subset \mathfrak{E}$ as the zero finite rank subbundle induces a $G$-equivariant $\mathbb{Z}_{2}$-graded $*$-homomorphism

$$
\Psi_{p}: \mathcal{A}(M) \rightarrow \mathcal{A}(\mathfrak{E}) .
$$

As discussed in the last section, we know that $\Psi_{p}$ induces an isomorphism on $K$ theory, but we want to show furthermore that $\Psi_{p}$ induces an isomorphism in equivariant $K$-theory. The main result of this paper is as follows.

Theorem 4.17. Let $G$ be a smooth, second countable, locally compact group and $M$ be a smooth isoreductive Riemannian $G$-manifold. If $p: \mathfrak{E} \rightarrow M$ is an affine Euclidean G-bundle with fiber a countably infinite dimensional Euclidean space then the inclusion of $M \subset \mathfrak{E}$ as the zero finite rank subbundle induces an isomorphism in equivariant $K$-theory:

$$
\Psi_{*}^{G}: K_{*}^{G}(\mathcal{A}(M)) \rightarrow K_{*}^{G}(\mathcal{A}(\mathfrak{E}))
$$

If the Euclidean space $\mathcal{E}$ is equipped with an orthogonal action of $G$, then we may consider the trivial Euclidean $G$-bundle $p: \mathcal{E} \rightarrow\{0\}$, where $M=\{0\}$ denotes the isoreductive (but very non-proper!) one-point Riemannian $G$-manifold consisting of the origin. Our theorem then reduces to the Bott periodicity theorem. The Thom map $\Psi_{p}=\beta$ is the "Bott map" in this case.

\section{Proof of the Thom Isomorphism Theorem 4.17}

Let $G$ be a smooth, second countable, locally compact, Hausdorff group.

Definition 5.1. A $G$ - $C^{\star}$-algebra $A$ is called isoreductive if there is a a second countable, locally compact, isoreductive metric $G$-space $(M, d)$ and a nondegenerate $G$-equivariant injection

$$
\theta: C_{0}(M) \rightarrow Z M(A)
$$


such that $\theta\left(C_{0}(M)\right) A$ is dense in $A$. If $f \in C_{0}(M)$ and $a \in A$ we write $f a=\theta(f) a$. If $A$ is $\mathbb{Z}_{2}$-graded then we assume that $\theta$ has grading degree zero, where $C_{0}(M)$ is trivially graded. That is, $A$ is a $\mathbb{Z}_{2}$-graded $C_{0}(M)$-algebra (Definition A.5) such that the structural homomorphism is $G$-equivariant.

If $A$ is an isoreductive $G$ - $C^{\star}$-algebra, and $M$ is the isoreductive metric space in the previous definition, then we will say that $A$ is isoreductive over $M$. For a discussion of the related notion of (ungraded) proper algebras, see [17].

Lemma 5.2. Let $A$ be a $\mathbb{Z}_{2}$-graded $G$ - $C^{\star}$-algebra. If $A$ is isoreductive over $M$ then $B \hat{\otimes} A$ is isoreductive over $M$ for any $\mathbb{Z}_{2}$-graded $G$ - $C^{\star}$-algebra $B$.

Example 5.3. Let $M$ be a second countable, isoreductive Riemannian $G$-manifold. The $\mathbb{Z}_{2}$-graded $G$-C $C^{\star}$-algebra $\mathcal{A}(M)=\mathcal{S} \hat{\otimes} \mathcal{C}(M)$ is isoreductive over $M$, where $\mathcal{S}$ has the trivial $G$-action.

Let $\mathcal{E}$ be a Euclidean space of countable dimension.

Proposition 5.4. Let $p: \mathfrak{E} \rightarrow M$ be an affine Euclidean $G$-bundle modelled on $\mathcal{E}$. The $C^{\star}$-algebra $\mathcal{A}(\mathfrak{E})$ is isoreductive over $M$.

Proof. If $E^{a} \subset \mathfrak{E}$ is a finite rank subbundle, then using the projection $p_{a}: E^{a} \rightarrow$ $M, \mathcal{A}\left(E^{a}\right)$ has a graded $C_{0}(M)$-algebra structure. Moreover, if $E^{a} \preceq E^{b}$ are finite rank subbundles, the Thom map $\mathcal{A}\left(E^{a}\right) \rightarrow \mathcal{A}\left(E^{b}\right)$ from Theorem 2.13 is clearly $C_{0}(M)$-linear. It follows by an approximation argument that the direct limit

$$
\mathcal{A}(\mathfrak{E})=\underset{a}{\lim _{a}} \mathcal{A}\left(E^{a}\right)
$$

has a graded $C_{0}(M)$-algebra structure and the induced action of $C_{0}(M)$ is $G$ equivariant.

If a $C^{\star}$-algebra $A$ is isoreductive over $M$, then $A$ is a $C_{0}(M)$-algebra and can thus be realized as the algebra of sections of an (upper-semicontinuous) $C^{\star}$-bundle over $M$ as follows $[\mathbf{1 5}, \mathbf{2 6}]$. Given $x \in M$, the fiber over $x$ is the quotient $A_{x}=A / I_{x}$ where $I_{x}$ is the ideal $I_{x}=C_{0}(M \backslash\{x\}) A$. For any $a \in A$, let $a(x)$ denote the image of $a$ in the fibre $A_{x}$. There is then a faithful representation of $A$ into the direct sum $\bigoplus_{x \in M} A_{x}$ given by $a \mapsto(a(x))_{x \in M}$.

Definition 5.5. (Compare Definition 2.1[15]) Let $A$ be isoreductive over $M$. Let $Y$ be a locally compact subset of $M$. The restriction of $A$ to $Y$ is defined as

$A_{Y}=C_{0}(Y) \cdot A=\left\{b \in \oplus_{y \in Y} A_{y}: b(y)=f(y) a(y)\right.$ for some $f \in C_{0}(Y)$ and $\left.a \in A\right\}$.

This definition makes sense for any $C_{0}(M)$-algebra (without grading or $G$-action.) The following is adapted from Lemma $2.2[\mathbf{1 5}]$.

Lemma 5.6. Let $A$ be isoreductive over $M$. Let $Y$ be a $G$-invariant locally compact subset of $M$. Then $A_{Y}$ is a $G-C_{0}(Y)$-algebra. If $U$ is a $G$-invariant open subset of $M$, then $A_{U}$ is a $G$-invariant ideal in $A=A_{M}$ and can be identified with $C_{0}(U) A$. Moreover, there is an exact sequence

$$
0 \longrightarrow A_{U} \longrightarrow A \longrightarrow A_{M \backslash U} \longrightarrow 0
$$

of $G$ - $C^{\star}$-algebras. 
Lemma 5.7. Let $A$ be isoreductive over $M$. If $U$ is a $G$-invariant open subset, then there is a cyclic six term exact sequence

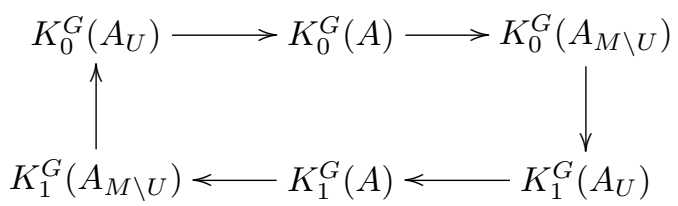

Proof. From the previous lemma, we have that

$$
0 \longrightarrow A_{U} \longrightarrow A \longrightarrow A_{M \backslash U} \longrightarrow 0
$$

is an exact sequence of $G-C^{\star}$-algebras. Thus, by the functorial properties of the full crossed product, we have an induced short exact sequence

$$
0 \longrightarrow A_{U} \rtimes G \longrightarrow A \rtimes G \longrightarrow A_{M \backslash U} \rtimes G \longrightarrow 0
$$

of crossed products. Now apply the $K$-theory six-term exact sequence [40].

The proof of the following result does not seem to be in the literature, but was communicated to the author by D. Williams.

Lemma 5.8. Let $A$ be a $G-C^{\star}$-algebra. Suppose that $I$ and $J$ are $G$-invariant ideals. Then $I \rtimes G$ and $J \rtimes G$ are ideals in $A \rtimes G$. Let $I \cap J$ denote the intersection ideal. Then,

$$
(I \rtimes G) \cap(J \rtimes G)=(I \cap J) \rtimes G .
$$

Proof. First recall that $I \rtimes G=\overline{C_{c}(G, I)}$ where the latter is viewed as an ideal of $C_{c}(G, A) \subset A \rtimes G$. Since

$$
C_{c}(G, I) \cap C_{c}(G, J) \subset C_{c}(G, I \cap J),
$$

we certainly have

$$
(I \rtimes G) \cap(J \rtimes G) \subset(I \cap J) \rtimes G .
$$

On the other hand, $(I \cap J) \rtimes G$ is the closure of

$$
\operatorname{span}\left\{f \otimes c: f \in C_{c}(G) \text { and } c \in I \cap J\right\},
$$

where $f \otimes c$ is the shorthand for the function $g \mapsto f(g) c$. By the Cohen factorization theorem [10] or an approximation argument, we can assume that $c=a b$ with $a \in I$ and $b \in J$. Now if $\left\{e_{i}\right\}$ is an approximate unit in $C_{c}(G)$, then

$$
\left(e_{i} \otimes a\right)(f \otimes b)=e_{i} * f \otimes c
$$

converges to $f \otimes c$ in $(I \cap J) \rtimes G$. It follows that we get equality in (7).

The following is an equivariant Mayer-Vietoris theorem.

Lemma 5.9. Let $A$ be isoreductive over $M$. If $U$ and $V$ are $G$-invariant open 
subsets of $M$, there is an exact sequence

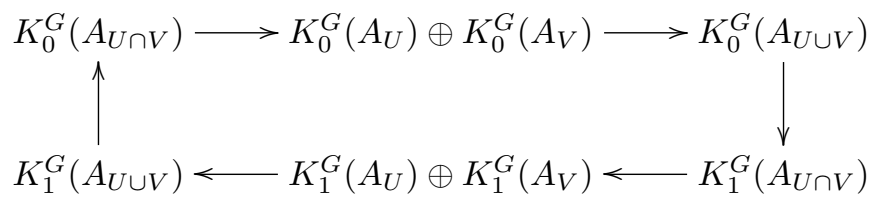

of equivariant $K$-theory groups.

Proof. Since $U$ and $G$ are $G$-invariant open sets, it follows that $A_{U}, A_{V}$, and $A_{U \cap V}$ are $G$-invariant ideals in $A_{U \cup V}$. We also have the $G$-equivariant identifications

$$
\left\{\begin{array}{l}
A_{U}+A_{V}=A_{U \cup V} \\
A_{U} \cap A_{V}=A_{U \cap V} .
\end{array}\right.
$$

It follows, using the previous lemma, that

$$
\left\{\begin{aligned}
A_{U} \rtimes G+A_{V} \rtimes G & =\left(A_{U}+A_{V}\right) \rtimes G & & =A_{U \cup V} \rtimes G \\
\left(A_{U} \rtimes G\right) \cap\left(A_{V} \rtimes G\right) & =\left(A_{U} \cap A_{V}\right) \rtimes G & & =A_{U \cap V} \rtimes G .
\end{aligned}\right.
$$

The result now follows from Definition 4.4 and Exercise 4.10.21 [20].

Lemma 5.10. Let $A$ be isoreductive over $M$. Suppose $M=\bigcup_{i \in I} U_{i}$ where $\left\{U_{i}\right\}_{i \in I}$ is an increasing directed system of $G$-invariant open subsets of $M$. That is, $U_{i} \subseteq U_{j}$ if $i<j$. Then we have isomorphisms

$$
\begin{aligned}
& A \cong \lim _{i \in I} \mathcal{A}_{U_{i}} \\
& K_{*}^{G}(A) \cong \lim _{i \in I} K_{*}^{G}\left(A_{U_{i}}\right) .
\end{aligned}
$$

Proof. The first direct limit isomorphism follows by an easy approximation argument. The $K$-theory isomorphism follows from the first and the fact that the $A_{U_{i}}$ are $G$-invariant ideals, which implies that there is a direct limit isomorphism

$$
A \rtimes G \cong \underset{i}{\lim } A_{U_{i}} \rtimes G
$$

of (full) crossed product $C^{\star}$-algebras. Now use the fact that $K$-theory is continuous, i.e., commutes with direct limits.

If $U \subset M$ is open, then for any finite rank subbundle $E^{a} \subset \mathfrak{E}$ we have that $\left.E^{a}\right|_{U} \subset E^{a}$ as an open subset and $\left.\left.E^{a}\right|_{U} \subset \mathfrak{E}\right|_{U}$ as a finite rank subbundle. Moreover, if $E^{a} \preceq E^{b} \preceq E^{c}$ then we have that $\left.\left.\left.E^{a}\right|_{U} \preceq E^{b}\right|_{U} \preceq E^{c}\right|_{U}$. Thus, we are led to make the following definition.

Definition 5.11. Let $p: \mathfrak{E} \rightarrow M$ be an affine Euclidean $G$-bundle. For any $(G$ invariant) open subset $i: U \hookrightarrow M$ we define the $C^{\star}$-algebra

$$
\mathcal{A}\left(\left.\mathfrak{E}\right|_{U}\right)=\operatorname{def}_{\underset{a}{\lim }} \mathcal{A}\left(\left.E^{a}\right|_{U}\right)
$$

where the direct limit is taken over all finite rank subbundles $E^{a} \subset \mathfrak{E}$. Note that we give the restricted bundle $\left.\mathfrak{E}\right|_{U}=i^{*}(\mathfrak{E})$ the connection $\nabla_{U}=i^{*}(\nabla)$. 
Lemma 5.12. Let $M$ be an isoreductive Riemannian $G$-manifold. Let $\mathfrak{E} \rightarrow M$ be an affine Euclidean $G$-bundle modelled on $\mathcal{E}$. If $U$ is an open subset of $M$, there are isomorphisms

$$
\begin{aligned}
\mathcal{A}(M)_{U} & \cong \mathcal{A}(U) \\
\mathcal{A}(\mathfrak{E})_{U} & \cong \mathcal{A}\left(\left.\mathfrak{E}\right|_{U}\right) .
\end{aligned}
$$

If $U$ is $G$-invariant, these isomorphisms are $G$-equivariant.

Proof. The first isomorphism follows from the definitions and the fact that $\mathcal{C}(M)_{U}$ $=C_{0}(U) \mathcal{C}(M)=\mathcal{C}(U)$. Given any finite rank subbundle $E^{a} \subset \mathfrak{E}$, it is easy to see that all elements in $\mathcal{A}\left(\left.E^{a}\right|_{U}\right)$ are in $\mathcal{A}(\mathfrak{E})_{U}$. This implies that the direct limit $\mathcal{A}\left(\left.\mathfrak{E}\right|_{U}\right) \subseteq \mathcal{A}(\mathfrak{E})_{U}$. For the reverse, it follows that $\mathcal{A}(\mathfrak{E})_{U} \cong \lim _{\longrightarrow} \mathcal{A}\left(E^{a}\right)_{U}$, but $\mathcal{A}\left(E^{a}\right)_{U} \subset \mathcal{A}\left(\left.E^{a}\right|_{U}\right)$ and so $\mathcal{A}(\mathfrak{E})_{U} \cong \mathcal{A}\left(\left.\mathfrak{E}\right|_{U}\right)$ as desired.

Before we can begin the proof, we need the following definition [34]. Let $H$ be a closed subgroup of $G$ and let $B$ be an $H$ - $C^{\star}$-algebra. Note that if $f: G \rightarrow B$ satisfies $f(g h)=h^{-1}(f(g))$ for all $g \in G$ and $h \in H$, then the function $g \mapsto\|f(g)\|$ is constant on cosets $g \cdot H$ and thus determines a well-defined function $\|f(\cdot H)\|$ on the quotient space $G / H$

Definition 5.13. Let $H$ be a closed subgroup of $G$ and let $B$ be an $H-C^{\star}$-algebra. The induced $C^{\star}$-algebra $\operatorname{Ind}_{H}^{G} B$ is defined as formula

$$
\operatorname{Ind}_{H}^{G} B=\left\{f \in C_{b}(G, B): f(g h)=h^{-1}(f(g)) \mathscr{E}\|f(\cdot H)\| \in C_{0}(G / H)\right\} .
$$

The group $G$ acts on $\operatorname{Ind}_{H}^{G} B$ by left translation: $(g f)(g)=f\left(g^{-1} g\right)$. A $\mathbb{Z}_{2}$-grading on $B$ induces a $\mathbb{Z}_{2}$-grading on $\operatorname{Ind}_{H}^{G} B$ in the obvious way.

For example, if $U$ is an $H$-space then $\operatorname{Ind}_{H}^{G} C_{0}(U) \cong C_{0}\left(G \times{ }_{H} U\right)$.

Lemma 5.14. Let $H$ be a closed subgroup of $G$. If $B$ is an $H-C^{\star}$-algebra, there are canonical isomorphisms

$$
K_{*}^{H}(B) \cong K_{*}^{G}\left(\operatorname{Ind}_{H}^{G} B\right) .
$$

Proof. By Green's Symmetric Imprimitivity Theorem [32], $\operatorname{Ind}_{H}^{G} B \rtimes G$ and $B \rtimes H$ are strongly Morita equivalent. Hence, they have the same $K$-theory and so:

$$
K_{*}^{H}(B)=K_{*}(B \rtimes H) \cong K_{*}\left(\operatorname{Ind}_{H}^{G} B \rtimes G\right)=K_{*}^{G}\left(\operatorname{Ind}_{H}^{G} B\right) .
$$

Proposition 5.15. Let $A$ be isoreductive over $M$. For any $x \in M$, let $U$ be an $H$-slice through $x$ as in Definition (4.1). There is a G-equivariant isomorphism

$$
\operatorname{Ind}_{H}^{G} A_{U} \cong A_{G U} .
$$

Hence, $K_{*}^{H}\left(A_{U}\right) \cong K_{*}^{G}\left(A_{G U}\right)$.

Proof. Since $U$ is $H$-invariant, it follows that the ideal $A_{U}$ is an $H$ - $C^{\star}$-algebra and has a $C_{0}(U)$-algebra structure. Similarly, $A_{G U}$ is a $G$ - $C_{0}(G U)$-algebra and isoreductive over $G U$. Thus, by Proposition 2.1 [26], there is a canonical continuous $G$-equivariant map (with dense range)

$$
\text { Res : Prim } A_{G U} \rightarrow G U
$$


where

$$
\operatorname{Res}(J)=x \Longleftrightarrow \overline{A_{G U} I_{x} A_{G U}}=C_{0}(G U \backslash\{x\}) A=A_{G U \backslash\{x\}} \subset J
$$

where $I_{x}=\left\{f \in C_{0}(M): f(x)=0\right\}$. Since $U$ is an $H$-slice, there is a $G$-equivariant homeomorphism

$$
\phi: G U \rightarrow G \times_{H} U .
$$

Let $p: G \times{ }_{H} U \rightarrow G / H$ be the $G$-map induced by the projection $G \times U \rightarrow G$. Thus, we have a canonical continuous $G$-equivariant map

$$
\sigma=p \circ \phi \circ \operatorname{Res}: \operatorname{Prim} A_{G U} \rightarrow G / H \text {. }
$$

Let $I$ be the ideal

$$
I=\bigcap\left\{J \in \operatorname{Prim} A_{G U}: \sigma(J)=e H\right\} .
$$

Note that $(p \circ \phi)^{-1}(e H)=U$. One can then check that $I=A_{G U \backslash U}$. By a theorem of Echterhoff $[\mathbf{1 3}, \mathbf{1 4}]$, there is a $G$-equivariant isomorphism

$$
\Phi: A_{G U} \rightarrow \operatorname{Ind}_{H}^{G} A_{G U} / I .
$$

Since $(p \circ \phi)^{-1}(e H)=U, U$ is a closed subset of $G U$. By Lemma 5.6:

$$
A_{G U} / I=A_{G U} / A_{G U \backslash U} \cong A_{U} .
$$

Thus, there is an induced isomorphism of crossed products

$$
\Phi^{G}: A_{G U} \rtimes G \rightarrow \operatorname{Ind}_{H}^{G} A_{U} \rtimes G
$$

and the result follows.

Let $\mathcal{E}$ be a Euclidean space of countably infinite dimension (with orthogonal $G$-action.)

Proposition 5.16. Let $\mathfrak{E}=M \times \mathcal{E} \rightarrow M$ be a trivial affine Euclidean $G$-bundle modelled on $\mathcal{E}$ (with the trivial $G$-connection). There is a $G$-equivariant isomorphism

$$
\mathcal{A}(\mathfrak{E}) \cong \mathcal{A}(\mathcal{E}) \hat{\otimes} \mathcal{C}(M)
$$

of $\mathbb{Z}_{2}$-graded $G$-C $C^{\star}$-algebras. Under this isomorphism, the Thom map has the form

$$
\Psi=\beta \hat{\otimes} \operatorname{id}_{\mathcal{C}(M)}: \mathcal{S} \hat{\otimes} \mathcal{C}(M) \rightarrow \mathcal{A}(\mathcal{E}) \hat{\otimes} \mathcal{C}(M)
$$

where $\beta: \mathcal{S}=\mathcal{A}(0) \rightarrow \mathcal{A}(\mathcal{E})$ is the Bott map of Lemma 3.12. Thus, the induced map $\Psi_{*}^{G}: K_{*}^{G}(\mathcal{A}(M)) \rightarrow K_{*}^{G}(\mathcal{A}(\mathfrak{E}))$ is an isomorphism on equivariant $K$-theory.

Proof. Let $\left\{V_{\alpha}\right\}$ be the collection of all finite dimensional subspaces of $\mathcal{E}$ ordered by subspace inclusion. Then $F^{\alpha}=M \times V_{\alpha}$ defines a directed system $\left\{F_{\alpha}\right\}$ of finite rank Euclidean subbundles such that $\mathfrak{E}=\bigcup_{\alpha} F^{\alpha}$. By Proposition 2.16 we have that

$$
\mathcal{A}\left(F^{\alpha}\right) \cong \mathcal{A}\left(V_{\alpha}\right) \hat{\otimes} \mathcal{C}(M) .
$$

Moreover, if $\alpha<\beta$ then the Thom map has the form

$$
\Psi_{\alpha \beta}=\beta_{\alpha \beta} \hat{\otimes} \operatorname{id}_{\mathcal{C}(M)}: \mathcal{A}\left(V_{\alpha}\right) \hat{\otimes} \mathcal{C}(M) \rightarrow \mathcal{A}\left(V_{\beta}\right) \hat{\otimes} \mathcal{C}(M) .
$$


This implies that we have a $*$-homomorphism

$$
\theta: \mathcal{A}(\mathcal{E}) \hat{\otimes} \mathcal{C}(M)=\underset{\alpha}{\lim _{\alpha}} \mathcal{A}\left(F^{\alpha}\right) \rightarrow \mathcal{A}(\mathfrak{E}) .
$$

Given any finite rank subbundle $E^{a} \subset \mathfrak{E}$, there is a $\beta$ such that $E^{a} \preceq F^{\beta}$. This implies that the directed subsystem $\left\{F^{\alpha}\right\}$ is cofinal with respect to the directed system $\mathcal{F} \mathcal{B}(\mathfrak{E})=\left\{E^{a}\right\}$. By Lemma L.1.5 $[\mathbf{4 0}]$, there is a $*$-homomorphism

$$
\mathcal{A}(\mathfrak{E}) \rightarrow \underset{\alpha}{\lim _{\alpha}} \mathcal{A}\left(F^{\alpha}\right) \cong \mathcal{A}(\mathcal{E}) \hat{\otimes} \mathcal{C}(M)
$$

which provides the inverse to $\theta$. If $E^{a} \preceq F^{\beta}$ then for any $g \in G$, we have that $g \cdot E^{a} \preceq g \cdot F^{\beta}=M \times g \cdot V_{\beta}=M \times V^{\delta}$ and so these isomorphisms are $G$ equivariant. Using the equivariant asymptotic morphism calculations in Sections 4, 5 and Appendix $\mathrm{C}$ of $[\mathbf{1 9}]$ we easily obtain (by tensoring with $\operatorname{id}_{\mathcal{C}(M)}$ ) the last statement.

Proof of Theorem 4.17. Using Definition 4.1, Mayer-Vietoris and Five Lemma arguments, direct limits, and transfinite induction, it suffices to show that the Thom map induces an isomorphism for $W=G U$, where $U$ is an $H$-slice for some closed subgroup $H$ of $G$. Since $U$ is $H$-equivariantly contractible, we may also assume that the restricted bundle $\left.\mathfrak{E}\right|_{U} \cong U \times \mathcal{E}$ is a trivial affine Euclidean $H$-bundle (with the trivial $H$-invariant connection.) By Proposition 5.16,

$$
\Psi_{*}^{H}: K_{*}^{H}(\mathcal{A}(U)) \rightarrow K_{*}^{H}\left(\mathcal{A}\left(\left.\mathfrak{E}\right|_{U}\right)\right)
$$

is an isomorphism of abelian groups. Now, by Lemma 5.14 and Proposition 5.15, it follows for $W=G U$ that

$$
\begin{array}{rlrl}
K_{*}^{G}(\mathcal{A}(W)) & \cong K_{*}^{G}\left(\operatorname{Ind}_{H}^{G} \mathcal{A}(U)\right) & & \\
& \cong K_{*}^{H}(\mathcal{A}(U)) & & \cong K_{*}^{H}\left(\mathcal{A}\left(\left.\mathfrak{E}\right|_{U}\right)\right) \\
& \cong K_{*}^{G}\left(\operatorname{Ind}_{H}^{G} \mathcal{A}\left(\left.\mathfrak{E}\right|_{U}\right)\right) & \cong K_{*}^{G}\left(\mathcal{A}\left(\left.\mathfrak{E}\right|_{W}\right)\right)
\end{array}
$$

and we are finished.

\section{Appendix A. $\mathbb{Z}_{2}$-Gradings and Unbounded Multipliers}

In this appendix we collect definitions and results on graded $C^{\star}$-algebras, tensor products, and unbounded multipliers needed in the text. Although some of these results may be found elsewhere $[\mathbf{5}, \mathbf{8}, \mathbf{1 9}, \mathbf{3 8}]$ we include them for completeness and to fix notation.

\section{A.1. Graded $C^{\star}$-algebras}

Recall that a $C^{\star}$-algebra $A$ is $\mathbb{Z}_{2}$-graded if it is equipped with a $*$-automorphism $\gamma \in \operatorname{Aut}(A)$ such that $\gamma^{2}=\mathrm{id}_{A}$. See Blackadar $[\mathbf{8}]$ for the basic theory and properties of graded $C^{\star}$-algebras.

Important examples for us are:

- The algebra $\mathcal{S}=C_{0}(\mathbb{R})$ of continuous functions on $\mathbb{R}$, vanishing at infinity, graded according to even and odd functions: $\gamma(f)(x)=f(-x)$. 
- The complex Clifford algebra $\operatorname{Cliff}(V)$ of a finite dimensional Euclidean vector space $V$ which is the unital $C^{\star}$-algebra generated by a real subspace $V$ of selfadjoint elements such that $v^{2}=\|v\|^{2}$ for all $v \in V$. The grading automorphism is induced by the map $\gamma: V \rightarrow V$ given by $\gamma(v)=-v$.

- Any $C^{\star}$-algebra can be given the trivial grading $\gamma=\mathrm{id}_{A}$. The $C^{\star}$-algebra $\mathbb{C}$ of complex numbers is always assumed to be trivially graded.

The graded commutator of two homogeneous elements $a, b \in A$ is defined as

$$
[a, b]=a b-(-1)^{\operatorname{deg}(a) \operatorname{deg}(b)} b a .
$$

We shall say that two elements of $A$ commute if their graded commutator is zero.

\section{A.2. Unbounded Multipliers}

Let $A$ be a $C^{\star}$-algebra. Recall that a multiplier of $A$ is a right $A$-module map $T: A \rightarrow A$ for which there exists an 'adjoint' right $A$-module map $T^{*}: A \rightarrow A$ such that

$$
\langle T x, y\rangle=\left\langle x, T^{*} y\right\rangle \quad \text { for all } x, y \in A,
$$

where the angle brackets denote the pairing

$$
\langle x, y\rangle=x^{*} y .
$$

Such multipliers form a $*$-algebra under composition, which is denoted $M(A)$. The operator norm of each multiplier $T$ is finite (by the Closed Graph Theorem), and with this norm $M(A)$ becomes a $C^{\star}$-algebra. It contains $A$ as an essential ideal. If $A$ has a $\mathbb{Z}_{2}$-grading, then $M(A)$ has an induced $\mathbb{Z}_{2}$-grading by the formula

$$
\operatorname{deg}(T x) \equiv \operatorname{deg}(T)+\operatorname{deg}(x)(\bmod 2)
$$

for all $T \in M(A)$ and $x \in A$. See the book [28] for more details.

We wish to consider some "unbounded" multipliers of $A$.

Definition A.1. (Compare [5, 11, 19].) An unbounded self-adjoint multiplier of a graded $C^{\star}$-algebra $A$ is an $A$-linear map $D: \mathcal{A} \rightarrow A$ from a dense, right $\mathbb{Z}_{2}$-graded $A$-submodule $\mathcal{A} \subset A$ into $A$ such that

(i) $\langle D x, y\rangle=\langle x, D y\rangle$, for all $x, y \in \mathcal{A}$; and

(ii) the operators $D \pm i I: \mathcal{A} \rightarrow A$ are isomorphisms.

(iii) $\operatorname{deg}(D x) \equiv \operatorname{deg}(x)+1(\bmod 2)$, for all $x \in \mathcal{A}$.

That is, $D$ has grading-degree one with respect to the gradings.

Remark A.2. The adjective 'unbounded' is conventional, but for brevity we shall often drop this term. Note that if $\mathcal{A}=A$ then the above definition produces a bounded operator.

\section{A.3. Essentially Self-Adjoint Multipliers}

If $\mathcal{A}$ is a graded, right $A$-submodule of $A$ and if $D: \mathcal{A} \rightarrow A$ satisfies

(i) $\langle D x, y\rangle=\langle x, D y\rangle$, for all $x, y \in \mathcal{A}$;

(ii) $\operatorname{deg}(D x) \equiv \operatorname{deg}(x)+1(\bmod 2)$, for all $x \in \mathcal{A}$; 
(iii) the operators $D \pm i I$ have dense range;

then the closure $\bar{D}$ of $D$ (in the sense of unbounded operator theory) is a self-adjoint multiplier. We shall call $D$ essentially self-adjoint and for simplicity we shall usually use the same symbol $D$ for the closure $\bar{D}$.

Here are some examples.

- Denote by $X$ the operator of "multiplication by $x$ " on $\mathbb{R}$, so that if $f$ is a function on $\mathbb{R}$ then $X f(x)=x f(x)$ for all $x \in \mathbb{R}$. Then $X$ is an unbounded selfadjoint multiplier of $\mathcal{S}=C_{0}(\mathbb{R})$, whose domain is the set of all functions $f \in \mathcal{S}$ such that $X f \in \mathcal{S}$.

- Let $V$ be a Euclidean vector space and let $\mathcal{C}(V)=C_{0}\left(V\right.$, Cliff $\left._{\mathbb{C}} V\right)$ be the $C^{\star}$ algebra of continuous functions from $V$ into the complexified Clifford algebra of $V$, with the induced grading. Denote by $C_{V}: V \rightarrow \operatorname{Cliff}_{\mathbb{C}}(V)$ the function which assigns to $v \in V$ the value $v \in \operatorname{Cliff}_{\mathbb{C}}(V)$. This defines an unbounded essentially self-adjoint multiplier of $\mathcal{C}(V)$ that is very useful in the formulation of equivariant Bott periodicity for infinite dimensional Euclidean spaces [19].

\section{A.4. Functional Calculus}

If $D$ is an unbounded (essentially) self-adjoint multiplier of $A$ then the resolvent operators $(D \pm i I)^{-1}: A \rightarrow A$ determine bounded multipliers of $A$. If $C_{b}(\mathbb{R})$ denotes the $C^{\star}$-algebra of bounded continuous functions on $\mathbb{R}$ then there is a unique *homomorphism (called the functional calculus of $D$ )

$$
\begin{gathered}
C_{b}(\mathbb{R}) \rightarrow M(A) \\
f \mapsto f(D),
\end{gathered}
$$

mapping the resolvent functions $(x \pm i)^{-1}$ to $(D \pm i I)^{-1}$. Compare [11]. This has the property that if $g(x)=x f(x)$ then $g(D)=D f(D)$. It also follows from the grading condition (iii) that the above $*$-homomorphism is grading preserving, if we grade $C_{b}(\mathbb{R})$ by even and odd functions.

\section{A.5. Graded Tensor Products}

We shall denote by $A \hat{\otimes} B$ the maximal graded tensor product of graded $C^{\star}$ algebras. It has the characteristic property that a pair of graded $*$-homomorphisms $A \rightarrow C$ and $B \rightarrow C$, whose images commute with one another, induces a morphism

$$
A \hat{\otimes} B \rightarrow C .
$$

In the instances we shall consider, this maximal tensor product is also equal to the minimal tensor product, defined with the help of representations of $A$ and $B$ on graded Hilbert spaces, since one of the factors will always be nuclear.

If $B$ is evenly graded, in the sense that there is a self-adjoint unitary $\varepsilon$ in $M(B)$ (the multiplier algebra of $B$ ) such that for all $b \in B$,

$$
\gamma(b)=\varepsilon b \varepsilon,
$$

then $A \hat{\otimes} B$ is isomorphic to the maximal tensor product of ungraded $C^{\star}$-algebras, $A \otimes B$, via the map

$$
a \hat{\otimes} b \mapsto a \otimes \varepsilon^{\operatorname{deg}(a)} b .
$$


This applies, for example, to $B=\mathcal{K}(H)$, where $H$ is a graded Hilbert space.

\section{A.6. Tensor products and multipliers}

Suppose that $D$ is an (essentially) self-adjoint multiplier of $A$. Then the operator $D \hat{\otimes} 1$, with domain the algebraic tensor product (over $\mathbb{C}$ ) $\mathcal{A} \hat{\otimes}_{\text {alg }} B$, is an essentially self-adjoint multiplier of $A \hat{\otimes} B$. Note that the functional calculus for $D \hat{\otimes} 1$ is given by $f(D \hat{\otimes} 1)=f(D) \hat{\otimes} 1$.

The following is stated in [19] (see also [5]), but the proof, which is due to $\mathrm{N}$. Higson, is included below.

Lemma A.3. Let $C$ and $D$ be essentially self-adjoint multipliers of $A$ and $B$. Then $C \hat{\otimes} 1+1 \hat{\otimes} D$ is an essentially self-adjoint multiplier of $A \hat{\otimes} B$, with domain the algebraic tensor product of the domains of $C$ and $D$.

Proof. Let us introduce for each $N>0$ the bounded multiplier

$$
D_{N}=D\left(1+N^{-2} D^{2}\right)^{-1 / 2}
$$

(and define $C_{N}$ similarly). Note that as $N \rightarrow \infty$ the function

$$
x_{N}=x\left(1+N^{-2} x^{2}\right)^{-1 / 2}
$$

converges, uniformly on compact sets, to the identity function $x \mapsto x$. So for $N$ large we expect $D_{N}$ to approximate $D$ somehow. In fact if $\phi$ is a compactly supported function then $D_{N} \phi(D) \rightarrow D \phi(D)$ in norm.

Let us make a further observation: for every $y \in A \hat{\otimes} B$ there is some compactly supported $\phi$ so that

$$
y \approx \phi(C) \hat{\otimes} \phi(D) y
$$

(in other words given $\varepsilon>0$ we can find $\phi$ so that the two sides are within $\varepsilon$ in norm).

Now given $y$ choose $\phi$ as above then choose $N$ so that $D_{N} \phi(D) \approx D \phi(D)$. By simple $C^{\star}$-algebra theory the bounded multiplier $C_{N} \hat{\otimes} 1+1 \hat{\otimes} D_{N}$ is essentially selfadjoint in the sense of 1.4. So there is some $x \in \mathcal{A} \hat{\otimes}_{\text {alg }} \mathcal{B}$ with

$$
y \approx\left(i I+C_{N} \hat{\otimes} 1+1 \hat{\otimes} D_{N}\right) x .
$$

But then

$$
\begin{aligned}
y \approx \phi(C) \hat{\otimes} \phi(D) y & \approx \phi(C) \hat{\otimes} \phi(D)\left(i I+C_{N} \hat{\otimes} 1+1 \hat{\otimes} D_{N}\right) x \\
& =\left(i I+C_{N} \hat{\otimes} 1+1 \hat{\otimes} D_{N}\right) \phi(C) \hat{\otimes} \phi(D) x \\
& \approx(i I+C \hat{\otimes} 1+1 \hat{\otimes} D) \phi(C) \hat{\otimes} \phi(D) x,
\end{aligned}
$$

so that $y$ is in the closure of the range of $(i I+C \hat{\otimes} 1+1 \hat{\otimes} D)$ as required.

Corollary A.4. If $C$ and $D$ are essentially self-adjoint multipliers of $A$ and $B$, then

$$
\exp \left(-(C \hat{\otimes} 1+1 \hat{\otimes} D)^{2}\right)=\exp \left(-C^{2}\right) \hat{\otimes} \exp \left(-D^{2}\right) .
$$




\section{A.7. $\quad$ Graded $C_{0}(M)$-algebras}

Let $M$ be a locally compact space. We assume that $C_{0}(M)$ has the trivial grading.

Definition A.5. $A C^{\star}$-algebra $A$ is called $a \mathbb{Z}_{2}$-graded $C_{0}(M)$-algebra if $A$ is $\mathbb{Z}_{2}$-graded and there is a $*$-homomorphism $C_{0}(M) \rightarrow Z M(A)$ of degree zero which is nondegenerate in the sense that $C_{0}(M) A$ is dense in $A$.

We now introduce a new tensor product, which is a graded version of the balanced tensor product considered by others $[\mathbf{9}, \mathbf{1 5}, \mathbf{3 4}]$.

Definition A.6. Let $A$ and $B$ be graded $C_{0}(M)$-algebras. We define the balanced (graded) tensor product $A \hat{\otimes}_{M} B$ to be the quotient of $A \hat{\otimes} B$ by the $\mathbb{Z}_{2}$-graded ideal $J_{M}$ generated by

$$
\left\{(f \cdot a) \hat{\otimes} b-a \hat{\otimes}(f \cdot b) \mid f \in C_{0}(M), a \in A, \text { and } b \in B\right\} .
$$

There is then a short exact sequence

$$
0 \longrightarrow J_{M} \longrightarrow A \hat{\otimes} B \stackrel{q_{M}}{\longrightarrow} A \hat{\otimes}_{M} B \longrightarrow 0
$$

of $\mathbb{Z}_{2}$-graded $C^{\star}$-algebras.

There is an isomorphism $C_{0}(M) \hat{\otimes}_{M} A \cong A$ given by the map $f \hat{\otimes} a \mapsto f \cdot a$.

This tensor product has the universal property that if $\phi: A \rightarrow C$ and $\psi: B \rightarrow C$ are graded $C_{0}(M)$-linear $*$-homomorphisms with the property that their ranges graded commute, then there is a unique graded $*$-homomorphism

$$
\phi \hat{\otimes}_{M} \psi: A \hat{\otimes}_{M} B \rightarrow M(C): a \hat{\otimes}_{M} b \mapsto \phi(a) \psi(b)
$$

where $a \hat{\otimes}_{M} b$ denotes the image of $a \hat{\otimes} b$ in $A \hat{\otimes}_{M} B$.

Proposition A.7. Let $M$ be a Riemannian manifold. If $E$ and $F$ are finite rank Euclidean bundles on $M$, then there is a $\mathbb{Z}_{2}$-graded $C_{0}(M)$-algebra isomorphism

$$
C_{0}(M, \operatorname{Cliff}(E \oplus F)) \cong C_{0}(M, \operatorname{Cliff}(E)) \hat{\otimes}_{M} C_{0}(M, \operatorname{Cliff}(F)) .
$$

Proof. There is an $\mathbb{Z}_{2}$-graded isomorphism of Clifford algebra bundles

$$
\operatorname{Cliff}(E \oplus F) \cong \operatorname{Cliff}(E) \hat{\otimes} \operatorname{Cliff}(F)
$$

which follows by Proposition 1.5 [21]. The result now follows by applying the same argument as used in Proposition $2.6[\mathbf{1 6}]$ and the universal property of the balanced tensor product.

Definition A.8. Let $p: E \rightarrow M$ be a continuous map of locally compact spaces. If $A$ is a $\mathbb{Z}_{2}$-graded $C_{0}(M)$-algebra, then we define the pullback $C^{\star}$-algebra $p^{*} A$ by the formula

$$
p^{*} A=C_{0}(E) \hat{\otimes}_{M} A
$$

where $C_{0}(E)$ is given a $C_{0}(M)$-algebra structure via $p^{*}: C_{0}(M) \rightarrow C_{b}(E) \cong$ $M\left(C_{0}(E)\right)$. Note that since $C_{0}(M)$ is trivially graded, this is the same definition as given in [15, 34]. 
The relationship to pullback vector bundles is contained in the next result.

Proposition A.9. Let E be a smooth finite rank Euclidean bundle on the Riemannian manifold $M$. If $p: N \rightarrow M$ is a smooth map of Riemannian manifolds, there is an isomorphism

$$
p^{*} C_{0}(M, \operatorname{Cliff}(E)) \cong C_{0}\left(N, \operatorname{Cliff}\left(p^{*} E\right)\right)
$$

of $\mathbb{Z}_{2}$-graded $C^{\star}$-algebras.

Proof. By universality, there is a pullback isomorphism of Clifford bundles $\operatorname{Cliff}\left(p^{*} E\right) \cong p^{*} \operatorname{Cliff}(E)$. The result now follows from Proposition $2.12[\mathbf{1 6}]$.

\section{A.8. Pushing Forward Multipliers}

Suppose we are given a $*$-homomorphism $\phi: A^{\prime} \rightarrow M(A)$ with the property that $A^{\prime} A$ is dense in $A$. Then there is an induced $*$ - homomorphism $M\left(A^{\prime}\right) \rightarrow M(A)$ extending this, characterized by $T\left(a^{\prime} a\right)=T\left(a^{\prime}\right) a$. See [28]. Similarly, an essentially self-adjoint multiplier $D^{\prime}$ of $A^{\prime}$, with domain $\mathcal{A}^{\prime} \subset A^{\prime}$, determines an essentially self-adjoint operator on $A$, which we shall denote $D$ here, by the formula

$$
D\left(a^{\prime} a\right)=\left(D^{\prime} a^{\prime}\right) a \quad\left(\text { where } a^{\prime} \in \mathcal{A}^{\prime} \text { and } a \in A\right) .
$$

Its domain is $\mathcal{A}^{\prime} A$. The functional calculus maps for the two operators $D^{\prime}$ and $D$ (or, to be more precise, for the closures of $D^{\prime}$ and $D$ ) are related by the following commutative diagram:

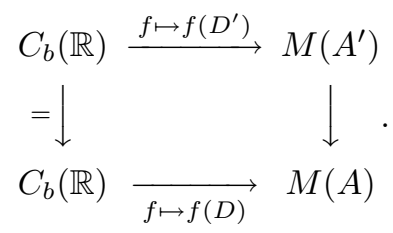

\section{References}

[1] H. Abels, Parallelizability of proper actions, global K-slices and maximal compact subgroups, Math. Ann. 212 (1974), 1-19.

[2] R. Abraham, J. E. Marsden, and T. Ratiu, Manifolds, Tensor Analysis, and Applications, 2nd. ed., Applied Mathematical Sciences, vol. 75, SpringerVerlag, New York, 1988.

[3] M. F. Atiyah, K-theory, W. A. Benjamin, New York, 1967.

[4] M. F. Atiyah, R. Bott, and A. Shapiro, Clifford modules, Topology 3 (1964), no. Suppl. 1, 3-38.

[5] S. Baaj and P. Julg, Théorie bivariante de Kasparov et opérateurs non bornés dans les $C^{*}$-modules Hilbertiens, C. R. Acad. Sci. Paris 296 (1983), 876-878, Se'rie I.

[6] P. Baum, A. Connes, and N. Higson, Classifying Space for Proper Actions and $K$-theory of Group $C^{*}$-algebras, Contemporary Mathematics 167 (1994), 241-291. 
[7] N. Berline, E. Getzler, and M. Vergne, Heat Kernels and Dirac Operators, Grundlehren der mathematischen Wissenschaften, vol. 298, Springer-Verlag, Berlin, 1986.

[8] B. Blackadar, K-theory for Operator Algebras, 2nd ed., MSRI Publication Series 5, Springer-Verlag, New York, 1998.

[9] E. Blanchard, Tensor products of $C(X)$-algebras over $C(X)$, Astérisque 232 (1995), 81-92.

[10] P. Cohen, Factorization in group algebras, Duke Math. J 26 (1959), 199-205.

[11] A. Connes, An Analogue of the Thom Isomorphism for Crossed Products of a $C^{*}$-Algebra by an Action of $\mathbb{R}$, Adv. in Math. 31 (1981), no. 1, 31-55.

[12] _ Noncommutative geometry, Academic Press, 1994.

[13] S. Echterhoff, On Induced Covariant Systems, Proc. Amer. Math. Soc. 108 (1990), no. 3, 703-706.

[14] Errata to "On Induced Covariant Systems", Proc. Amer. Math. Soc. 116 (1992), no. 2, 581.

[15] S. Echterhoff and D. P. Williams, Crossed Products by $C_{0}(X)$-actions, J. Funct. Anal. 158 (1998), 113-151.

[16] J. M. Gracia-Bondía, J. C. Varilly, and H. Figueroa, Elements of Noncommutative Geometry, Birkhäuser Advanced texts, Birkhäuser, Boston, Basel, Berlin, 2001.

[17] E. Guenter, N. Higson, and J. Trout, Equivariant E-theory for $C^{*}$-algebras, Memoirs of the AMS, vol. 148, no. 703, Amer. Math. Soc., Providence, R. I., 2000.

[18] N. Higson and G. Kasparov, E-theory and K-theory for groups which act properly and isometrically on Hilbert space, Invent. Math. 144 (2001), 23-74.

[19] N. Higson, G. Kasparov, and J. Trout, A Bott Periodicity Theorem for Infinite Dimensional Euclidean Space, Advances in Mathematics 135 (1998), no. 1, $1-40$.

[20] N. Higson and J. Roe, Analytic K-Homology, Oxford Mathematical Monographs, Oxford University Press., Oxford, England., 2000.

[21] H. B. Lawson Jr. and M-L Michelsohn, Spin Geometry, Princeton University Press, Princeton, 1989.

[22] M. Karoubi, K-theory, An Introduction, Grundlehren der Mathematischen Wissenschaften, vol. 226, Springer-Verlag, New York, 1978.

[23] G. G. Kasparov, An Index for invariant elliptic operators, K-theory, and representations of Lie groups, Soviet Math. Dokl. 27 (1983), no. 1, 105-109.

[24] N. H. Kuiper, The homotopy type of the unitary group of Hilbert space, Topology 3 (1965), 19-30.

[25] S. Lang, Introduction to Differentiable Manifolds, John Wiley \& Sons, Inc., New York, 1962.

[26] M. Nilsen, $C^{\star}$-bundles and $C_{0}(X)$-algebras, Indiana Univ. Math. J. 45 (1996), $464-477$. 
[27] A. T. Paterson, The analytic index for proper, Lie groupoid actions, Contemp. Math. 282 (2001), 115-135.

[28] G. K. Pedersen, $C^{*}$-algebras and their Automorphism Groups, London Mathematical Society Monographs, vol. 14, Academic Press, London, 1979.

[29] N. C. Phillips, Equivariant K-theory and freeness of group actions on $C^{*}$ algebras, Springer-Verlag Lecture Notes in Mathematics, no. 1274, SpringerVerlag, Berlin, 1987.

[30] _ Equivariant K-theory for proper actions, Pitman Research Notes in Mathematics, vol. 178, Longman Scientific and Technical, 1989.

[31] R. Plymen, Strong Morita Equivalence, Spinors and Symplectic Spinors, J. Operator Theory 16 (1986), 305-324.

[32] I. Raeburn and J. Rosenberg, Crossed products of continuous-trace $C^{\star}$ algebras by smooth actions, Trans. Amer. Math. Soc, 305 (1988), 1-45.

[33] I. Raeburn and D. P. Williams, Pull-backs of $C^{\star}$-algebras and crossed products by certain diagonal actions, Trans. Amer. Math. Soc, 287 (1985), 755-777.

[34] Morita Equivalence and Continuous-Trace $C^{\star}$-Algebras, Mathematical Surveys and Monographs, vol. 60, American Mathematical Society, 1998.

[35] M. A. Rieffel, Morita equivalence for operator algebras, Proc. Symp. Pure Math 38 (1982), 285-298.

[36] G. Segal, Equivariant K-theory, Publ. Math. I.H.E.S. 34 (1968), 129-151.

[37] J. Trout, Asymptotic Morphisms and Elliptic Operators over $C^{*}$-Algebras, $K$-Theory 18 (1999), 277-315.

[38] - On Graded k-theory, Elliptic Operators and the Functional Calculus, Illinois Journal of Mathematics 18 (1999), 277-315.

[39] S. Wassermann, Exact $C^{*}$-Algebras and related topics, Seoul National University Research Institute of Mathematics Global Analysis Research Center, Seoul, South Korea, 1994.

[40] N. E. Wegge-Olsen, $K$-theory and $C^{*}$-algebras, Oxford University Press, New York, 1993.

This article may be accessed via WWW at http://www.rmi.acnet.ge/hha/ or by anonymous ftp at

ftp://ftp.rmi.acnet.ge/pub/hha/volumes/2003/n1a7/v5n1a7.(dvi,ps,pdf)

Jody Trout jody.trout@dartmouth.edu

6188 Bradley Hall

Dartmouth College

Hanover, NH 03755 